\title{
The Ebbinghaus illusion modulates visual search for size-defined targets: Evidence for preattentive processing of apparent object size
}

\author{
ASTRID BUSCH and HERMANN J. MÜLLER \\ Ludwig-Maximilians-Universität München, Munich, Germany
}

\begin{abstract}
Five search experiments investigated whether the apparent size of objects is, like their retinal size, coded in preattentive vision. Observers searched for a target circle that was either larger or smaller than distractor circles, with both types of test circles surrounded by context circles modulating apparent size (i.e., the Ebbinghaus illusion). The size ratio between the test and the context circles was manipulated in such a way that the test circles were surrounded by, for example, smaller context circles (making the larger target appear even larger) or by larger context circles (making the smaller distractors appear even smaller). Under optimal conditions, the detection reaction times were independent of the number of test circles in the display, and the Ebbinghaus illusion facilitated the detection of the target even in comparison with control conditions without any context circles. This finding is consistent with preattentive, spatially parallel processing of apparent size.
\end{abstract}

Treisman and her colleagues (Treisman, 1988; Treisman \& Gelade, 1980) proposed a model of visual search that conceives of visual processing as a two-stage process, with an early preattentive stage followed by a later attention-dependent stage. A target object differing in a distinctive visual attribute, or feature, from the distractor objects in the search display is searched for in parallel. The time taken to discern its presence - that is, the search reaction time (RT) - does not increase markedly with increasing numbers of objects in the display (i.e., the display size), and the target is experienced as "popping out" of the display. Target detection is thought to be mediated by a unique activation in a map of feature detectors tuned to the particular (feature) value the target takes on within its defining (feature) dimension. It is known that visual search for a variety of feature targets can operate efficiently, including targets defined by orientation, color, motion, (stereoscopic) depth, and, most important in the present context, size (see Wolfe, 1998, for a review). Studies that have demonstrated efficient search for sizedefined targets (e.g., Müller, Heller, \& Ziegler, 1995) have given rise to the notion of parallel preattentive processing of object size.

In contrast to search for distinctive feature targets, search for a target differing from the distractors by a con-

The authors thank the reviewers for their helpful suggestions on earlier versions of the manuscript. This research was supported by a grant from the Deutsche Forschungsgemeinschaft (MU 773/2-3). Correspondence concerning this article should be addressed to A. Busch, Department Psychologie, Ludwig-Maximilians-Universität München, Leopoldstrasse 13, D-80802 München, Germany (e-mail: abusch@) psy.uni-muenchen.de).

Note-This article was accepted by the previous editorial team, headed by Neil Macmillan. junction of features defined within different dimensions (e.g., orientation and color) is less efficient, with search RTs increasing linearly with increasing display size. Treisman and Gelade (1980) interpreted such search RT functions as reflecting serial, object-by-object search. However, subsequent findings that different types of conjunction search produce RT functions with slopes ranging from shallow to steep have led to the conception of search efficiency in terms of a continuum (rather than the parallel-serial dichotomy), and several theories have been advanced to explain how this continuum is generated (e.g., Cave \& Wolfe, 1990; Duncan \& Humphreys, 1989; Pashler, 1987; Treisman \& Sato, 1990).

In a number of experiments, Treisman and Gormican (1988) have demonstrated search asymmetries. In their studies, search was more efficient when observers searched for a target defined by the presence or greater value of a special attribute than when observers searched for a target defined by the absence or lesser value of the same attribute. For example, for size-defined targets, Treisman and Gormican obtained shallower RT function slopes when search was for a large target in a display of smaller distractors than when search was for a small target in a display of larger distractors. Furthermore, they found slopes that were shallower for size-defined targets that were easier to discriminate than for those that were more difficult to discriminate. Found (1997) confirmed their results for size-defined targets.

Thus, the presence of a differently sized target object in a search display can be discerned efficiently, in parallel, provided that the target is bigger than the distractors and the size difference between them is sufficiently large. It is, thus, clear that size information is derived and represented in some form in preattentive vision. However, it remains an open issue whether not only the image-based 
(retinal) size but also the perceived (apparent) size of objects is computed preattentively. The distinction between retinal and apparent object size refers to the phenomenon that, for example, an object of a given retinal size that is perceived to be farther from the observer appears larger than an object of the same retinal size perceived to be nearer. That is, the apparent size of an object takes into account its perceived distance from the observer. Most studies that have investigated search for size targets have used simple two-dimensional displays. In the relationship between retinal and apparent size, this represents a special case. Since two-dimensional displays contain no depth information, observers in these studies would have assumed that all of the items were at the same distance. Thus, any differences in apparent size between items would have been entirely concomitant with differences in retinal size.

However, in several recent studies (Aks \& Enns, 1996; Found \& Müller, 2001; Humphreys, Keulers, \& Donnelly, 1994), the apparent size of the display objects was manipulated by presenting them on a background texture gradient that provided secondary depth cues. If the apparent size of the display object is influenced by this scenic context, it should be easier to detect a size-defined target (e.g., the largest object) when its retinal size difference relative to the distractor objects is consistent with its apparent size (i.e., the large target object positioned farther from the observer). In agreement with this hypothesis, Aks and Enns reported effects of target position that varied according to whether the target object was smaller or larger than the distractor objects: Small near and large far targets were responded to more quickly than were small far and large near targets. These effects are explicable in terms of the apparent size of a target object reinforcing its retinal size. Although some evidence that the apparent size of display objects is derived and represented preattentively was provided in that study, the results were not entirely conclusive. In particular, the apparent size manipulation did not influence the slope of the search RT functions (as the apparent size difference between the target and distractor objects increased, slopes should have decreased), but rather influenced their $y$-intercept. It is possible that observers searched for the target object on the basis of retinal size differences, deriving apparent size information and modulating responses after having localized the target (i.e., not preattentively). Similar problems beset the findings of Humphreys et al. (1994) and Found and Müller (2001). Search rate differences would have been expected in their studies if the discriminability of the target relative to the distractor objects had been altered by the depth manipulation. This would have increased search efficiency in consistent conditions, in which the positioning of the display objects supported the size contrast between the target and the distractors (e.g., large far target among small near distractors) and decreased it in inconsistent conditions (see Found \& Müller, 2001, for a detailed discussion).

Therefore, in the present study we reinvestigated the representation of apparent size in preattentive vision.
However, the experiments, rather than being designed to use secondary depth cues to manipulate the apparent size of display objects, instead were designed to exploit a geometric optical illusion of apparent size: that produced by Ebbinghaus configurations. Such figures, described by Ebbinghaus (1913), consist of an inner (test) circle surrounded by several (context) circles. Smaller context circles make the test circle appear larger in comparison with an identical control circle without any context circles. In contrast, a test circle surrounded by larger context circles appears smaller. Psychophysical studies of this illusion have shown that the extent of misjudgment of the test circle increases with an increase in the size difference between test and context circles (Massaro \& Anderson, 1971). But these effects decrease, or even disappear, when context and test circles are presented successively (Cooper \& Weintraub, 1970; Jaeger \& Pollack, 1977).

One account of the Ebbinghaus apparent size illusion assumes that the test and context circles are perceived to lie in different depth planes. Depth-based interpretations have been shown to play a role with stereoscopically presented Ebbinghaus configurations. For example, Coren (1971) reported that context circles positioned farther from the observer were perceived as larger than were the context circles positioned closer. Furthermore, test circles surrounded by context circles of the same retinal size as the test circle but positioned in different depth planes were perceived as differentially large: Test circles surrounded by more distant (i.e., larger) context circles were perceived as smaller than were test circles surrounded by closer (i.e., smaller) context circles. However, in a study that compared monocularly visible with purely cyclopean Ebbinghaus configurations, Papathomas, Feher, Julesz, and Zeevi (1996) showed that "the [apparent size] illusion is clearly stronger for monocularly visible stimuli than for the cyclopean stimuli, but the effect of depth [i.e., differential retinal disparity between test and context circles] is small for [monocularly visible] objects.... The illusion is strongest when test and [context] circles are at or near the same depth, and its strength decreases as the depth difference increases" (pp. 787-788). This suggests that a depth-based interpretation of the configurations contributes little to the Ebbinghaus apparent size illusion (and that an account in terms of size contrast is more appropriate).

In the present (visual search) experiments, observers were presented with displays consisting of varying numbers of Ebbinghaus configurations (there were also control conditions in which only the inner, or test, circles of the Ebbinghaus configurations were displayed). The target to be detected was a test circle (surrounded by context circles) that was, for example, larger than the distractor test circles (surrounded by context circles). If the apparent size of the target and distractor test circles is derived and represented preattentively, spatially in parallel (like the retinal sizes of the target and distractor test circles), then target detection should be facilitated, relative 
to the control condition, by the context circles, which reinforce the retinal size difference between the target and distractor test circles, with the facilitatory effect being independent of display size. If the context circles make the target test circle appear substantially larger (or smaller) and if observers can exploit this illusion, then target detection might be successful even if the target and distractor test circles without any context circles are hard to discriminate on the basis of their retinal size alone (difficult search task). If the presence of context circles interferes with the processing of the target and distractor test circles and if this interference can be reduced by, for instance, advance presentation of the context circles, visual search in the experimental conditions should be similar to that in the control conditions. These hypotheses were tested in five experiments.

\section{EXPERIMENT 1}

Experiment 1 was designed to examine whether efficient search for a size-defined target test circle among distractor test circles (characterized by flat search RT functions) is enhanced by the context circles surrounding the test circles; that is, whether efficient search for a target test circle retinally larger or smaller than the dis- tractor test circles is sensitive to the apparent size modulation of the test circles by the context circles. There were two types of context circle conditions: context circle size consistent and inconsistent with the search instruction (see Figure 1). In the consistent conditions, observers searched for a target larger than the distractors, with all test circles surrounded by smaller context circles, or for a target smaller than the distractors, with test circles surrounded by larger context circles. That is, the context circle size was consistent with the search instruction, because the target test circle remained the largest or smallest stimulus in the display. In the inconsistent conditions, observers searched for a target larger than the distractors, with all test circles surrounded by larger context circles, or for a target smaller than distractors, with test circles surrounded by smaller context circles. That is, the context circle size was inconsistent with the search instruction, because the target test circle was no longer the largest or smallest stimulus in the display.

In both conditions, it was expected that, if search performance is sensitive to the apparent size modulation, target detection should be enhanced by the context circles, which reinforce the size difference between the target and distractor test circles. For instance, in the large target condition, although smaller context circles (i.e.,
Control condition

\begin{abstract}
Large target
\end{abstract}
condition

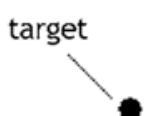

Small target

condition
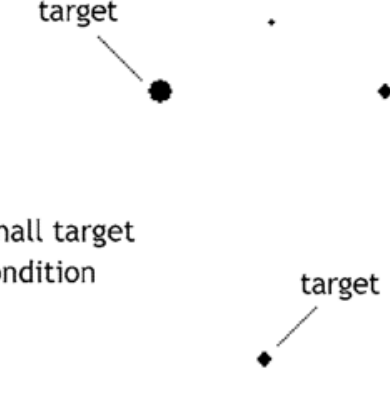

distractor

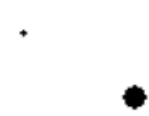

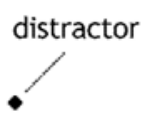

Consistent condition
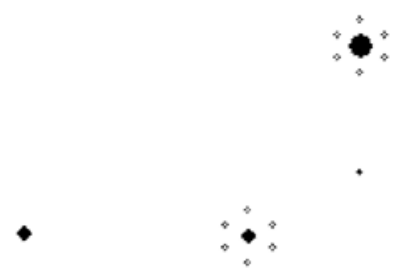

$\cdot$
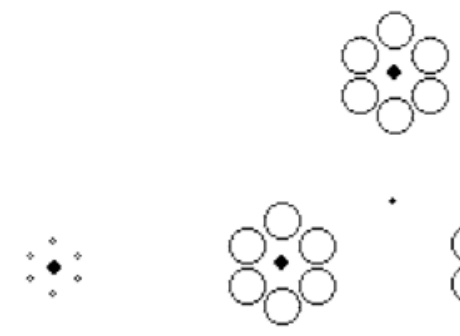

Inconsistent condition
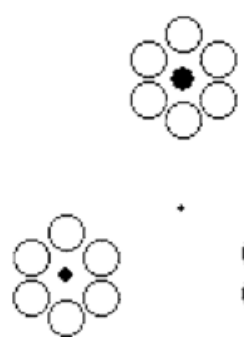

$+$

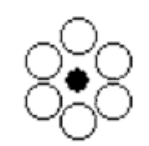

$+$

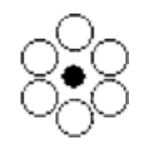

$-2$

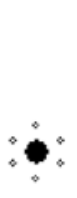

$+$
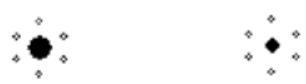

Figure 1. Examples of displays for control, consistent, and inconsistent search conditions for the small and large target conditions, respectively. With search for large targets, smaller context circles were presented in consistent conditions and larger context circles were presented in inconsistent conditions. By contrast, with search for small targets, larger context circles were presented in consistent conditions and smaller context circles were presented in inconsistent conditions. In both consistent and inconsistent conditions, the apparent size modulation was expected to reinforce the retinal size difference between the target and distractor test circles in line with the instructed direction of the search in consistent conditions and against the instructed direction in inconsistent conditions. 
consistent conditions) would make both the target and the distractor test circles appear larger, the increase in apparent size would be greater for the target (because of the larger size contrast between the target test and its surrounding context circles; Massaro \& Anderson, 1971), thereby increasing the apparent size difference between the target and the distractors and expediting target detection. Larger context circles in the large target condition (i.e., inconsistent conditions) would make both the target and the distractor test circles appear smaller, but the apparent size modulation would be stronger for the distractors (because of the larger size contrast between the distractor test and the surrounding context circles), again increasing the apparent size difference between the target and distractors.

However, it may be more difficult to exploit the apparent size modulation in inconsistent conditions, because the target is intermediate in size between the context and the distractor test circles, with search for "in-between" targets being especially hard (e.g., Treisman \& Gelade, 1980; Wolfe, 1994, 1998). Consequently, the increased search difficulty under inconsistent conditions may outweigh any facilitation produced by the Ebbinghaus apparent size illusion. The effects of context circles were assessed against a control condition in which target and distractor test circles were presented on their own (i.e., without any context circles). At least the consistent conditions were expected to produce benefits, relative to the control condition. If the apparent size illusion is coded preattentively and in parallel, at least the benefits were expected to be independent of display size.

\section{Method}

Participants. Fourteen Leipzig University students (11 female; ages ranged from 19 to 26 years) participated as observers in a single experimental session. All had experience with visual search tasks. All reported normal or corrected-to-normal vision. The observers were paid or received course credit for their participation.

Apparatus. The displays were presented on an AcerView 77e monitor driven by a personal computer. The laboratory was dimly illuminated. RTs were measured from the onset of the test circles. The responses were recorded from a mouse (right button for targetpresent response; left button for target-absent response) with the tracking ball removed to improve the accuracy of timing (Segalowitz \& Graves, 1990). The observers viewed the display from a distance of $60 \mathrm{~cm}$, which was maintained by the use of a chinrest.

Stimuli. The stimulus displays consisted of filled black test circles $\left(0.40 \mathrm{~cd} / \mathrm{m}^{2}\right)$ and unfilled context circles (with black outline) presented on a white background $\left(17.0 \mathrm{~cd} / \mathrm{m}^{2}\right)$. The observers had to decide whether one of the black test circles (target test circle) was larger or smaller than were the other black circles (distractor test circles). On half of the trials, the target test circle was present in the display, with target position varying randomly across trials.

The target test circle was either $10 \mathrm{~mm}$ and the distractor test circles $6 \mathrm{~mm}$, or vice versa. In the control conditions, only the target and distractor test circles were presented (i.e., there were no context circles). In the experimental conditions, each test circle was surrounded by six context circles that were equidistant from the test circle and from each other. The context circles were either 3 or $16 \mathrm{~mm}$ in diameter, and all were equal in size in a given search display. ${ }^{1}$ For a given size of context circle, the center-to-center distances between the test and the context circles were equivalent (13 and
$21 \mathrm{~mm}$ for small and large context circles, respectively; since context circles were equally sized in a given trial display, the center-tocenter distances between the test and the context circles were equivalent for all configurations within the display). For a given test circle size, the perimeter-to-perimeter distances between the test and context circles were equivalent (10 and $8 \mathrm{~mm}$ for small and large test circles, respectively). The shorter center-to-center distances for small context circles than for large context circles were introduced to ensure that the context circles were perceptually grouped with the test circles (forming an Ebbinghaus configuration), whereas the greater distances with large context circles were introduced to avoid overlap between the context circles and between the context and test circles. ${ }^{2}$ By manipulating the size difference between target test and target context circles, both consistent and inconsistent search conditions were realized.

Each search display (see Figure 1 for examples) consisted of a central fixation cross and three, five, or seven configurations of test circles surrounded by context circles. The configurations were positioned on an imaginary circle (with a radius of $70 \mathrm{~mm}$ ) around the center of the screen, with equal separations between the configurations. The number of configurations was limited to a maximum of seven, in order to avoid overlapping of the configurations (with numbers greater than seven, the configurations would have overlapped, at least with large context circles). The overall display dimensions were $20^{\circ}$ of visual angle in height $\times 20^{\circ}$ in width.

Design and Procedure. The independent variables in Experiment 1 were as follows: size of target test circle (small or large), display size (three, five, or seven Ebbinghaus configurations), target (present or absent), and size of context circles (zero [i.e., no context circles], small, or large).

An experimental session consisted of 900 test trials (25 trials for each of the 36 sizes of target circles $\times$ display size $\times$ target $\times$ size of context circles conditions) and lasted approximately $1 \mathrm{~h}$. The session was subdivided into 18 blocks of 50 trials. In half of the blocks, the target test circle was larger than the distractor test circles; in the other half, the target test circle was smaller. The order of target size blocks was counterbalanced across observers. The display size was kept constant throughout each block but varied across blocks. The size of the context circles was varied within each block. Each block started with six (unrecorded) practice trials (one trial for each of the six different trial types presented within the block). The observers initiated a block by pressing one of the mouse bottoms.

Each trial began with the presentation of a small black cross in the center of the screen, which the observers were instructed to fixate and around which the display elements would appear later. The display elements were presented $750 \mathrm{msec}$ after the fixation cross and remained visible until the observer gave a response. After an incorrect response, the observer received an acoustic feedback signal (beep) for $200 \mathrm{msec}$. Following a correct response, the screen went blank (white) for an intertrial interval of $750 \mathrm{msec}$; following an incorrect response, the interval was increased to $1,750 \mathrm{msec}$. Then the next trial began, unless the end of a block had been reached.

The observers were instructed to respond target-present or targetabsent as quickly and as accurately as possible. Before each halfsession, the observers were informed about the upcoming target condition (search for a target test circle smaller or larger than the distractor test circles).

\section{Results}

RT analysis. For each observer, mean RTs and associated standard deviations ( $S D$ s) were calculated for each experimental condition. Next, RTs outside the range of $\pm 2.5 \mathrm{SD}$ s from the mean were eliminated as outliers before the mean RTs were recalculated for further data analysis. This procedure produced a loss of $4.6 \%$ of the data. 
RT analysis was restricted to target-present trials. For target-absent trials, no differential effects of the context circles and, therefore, no apparent size modulations were expected. Figure 2 presents the group mean correct targetpresent RTs as a function of display size for the small and large target (test circle) conditions, respectively. Table 1 presents a summary of the search rates and base RTs (i.e., the slopes and $y$-intercepts of the search RT functions).

As can be seen from Figure 2, target-present RTs were differentially modulated by the context circles in the large (but not the small) target condition: Relative to the control condition, RTs were expedited across display size in the consistent condition (small context circles) and slowed in the inconsistent condition (large context circles).

The observers' mean correct target-present RTs were examined by a repeated measures analysis of variance (ANOVA), with size of target test circle (small or large), display size (three, five, or seven), and size of context circles (zero, small, or large) as factors. All main effects were revealed to be significant $[F(1,13)=21.137, p<$ .001 , for size of target test circles; $F(2,26)=9.217, p<$ .004 , for display size; and $F(2,26)=36.597, p<.001$, for size of context circles]. Furthermore, the size of target test circle $\times$ display size interaction $[F(2,26)=12.315$, $p<.001]$ and the size of target test circle $\times$ size of context circles interaction $[F(2,26)=41.255, p<.001]$ were significant. Target-present RTs were slower when the observers searched for a target test circle that was smaller than the distractor test circles than when they searched for a target test circle that was larger than the distractor test circles (i.e., significant search asymmetry). Overall, RTs increased with an increase in the size of the context circles and were affected by display size. However, display size significantly influenced target detection when the observers searched for a small target test circle, but not when they searched for a large one (size of target test circle $\times$ display size interaction). Furthermore, the significant interaction between size of target test circle and size of context circles indicated that the various sizes of context circles had differential effects on search performance in the two consistency conditions.

This interaction was explored further by two ANOVAs conducted separately for the two target size conditions (small and large), with display size (three, five, or seven) and size of context circles (zero, small, or large) as factors. These analyses revealed a significant main effect of display size when the target test circle was smaller than the distractor test circles $[F(2,26)=23.554, p<.001]$, but no main effect of display size when the target test circle was larger $[F(2,26)=0.336, p=.721]$. When the participants searched for a small target test circle, RTs increased significantly with increasing display size (about $5.4 \mathrm{msec} / \mathrm{item}$ ), but when they searched for a large target test circle, the search functions remained flat (about $0.5 \mathrm{msec} /$ item).

Overall, detection RTs were influenced by whether or not the test circles were surrounded by context circles and by the size of context circles. Comparisons between the consistent and inconsistent search conditions showed significantly faster RTs for consistent displays when the target test circle was large (i.e., faster RTs when the target and distractor test circles were surrounded by smaller, rather than larger, context circles) for all display sizes [combined across display size, the RT difference was $32 \mathrm{msec} ; t(13)=-5.812,-8.088$, and -4.914 for display sizes of three, five, or seven, respectively; all $p \mathrm{~s}<$ $.001]$. But there were no significant differences between consistent and inconsistent conditions when the target test circle was small [across display size, the difference was $0 \mathrm{msec} ; t(13)=-0.692,0.515$, and 0.519 , respectively].

For the large target test circle, smaller context circles increased the perceived target-distractor difference, making the target test circle appear even larger and, thus, easier to detect among the distractor test circles. Indeed, in two of the three display size conditions, the presence of smaller context circles significantly expedited the detection of a large target even relative to the control con-

\section{A Large target test circle} Target present

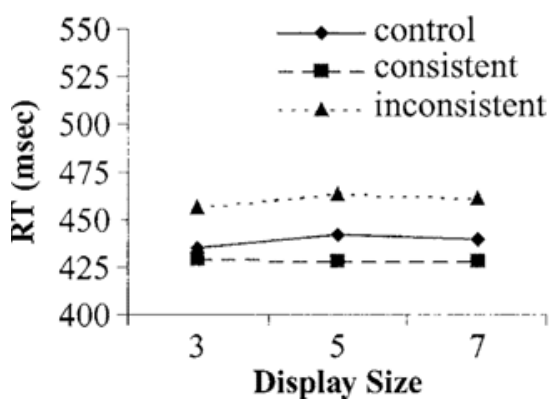

\section{B Small target test circle} Target present

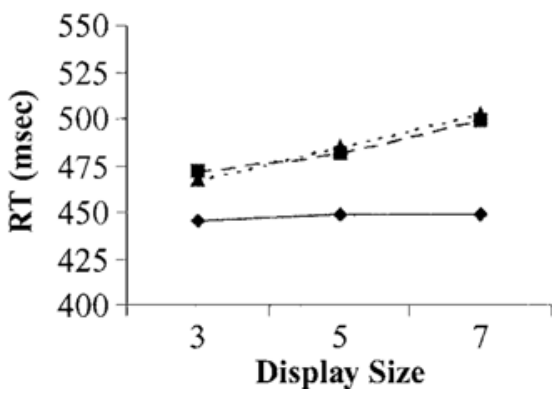

Figure 2. Mean target-present search reaction times (RTs, in milliseconds) in Experiment 1 for (panel A) large and (panel B) small target test circles. Control, consistent, and inconsistent conditions are represented by solid, broken, and dotted lines, respectively. 
Table 1

Base Reaction Times (in Milliseconds), Search Rates (in Milliseconds/Item), and Miss and False Alarm Rates (in Percentages) as a Function of Display Size, Separately for the Large and Small Target Conditions and the Context Conditions (Control, Consistent, and Inconsistent) in Experiment 1

\begin{tabular}{|c|c|c|c|c|c|c|c|c|c|c|}
\hline \multirow{2}{*}{$\begin{array}{c}\text { Context } \\
\text { Condition }\end{array}$} & \multicolumn{2}{|c|}{ Base } & \multicolumn{2}{|c|}{ Rate } & \multicolumn{3}{|c|}{ Misses } & \multicolumn{3}{|c|}{ False Alarms } \\
\hline & Present & Absent & Present & Absent & 3 & 5 & 7 & 3 & 5 & 7 \\
\hline \multicolumn{11}{|c|}{ Large Target } \\
\hline Control & 433 & 452 & 1.07 & 0.55 & 3.42 & 2.85 & 3.71 & 3.71 & 2.85 & 1.42 \\
\hline Consistent & 430 & 489 & -0.41 & -2.57 & 2.28 & 0.85 & 1.14 & 3.71 & 2.57 & 3.14 \\
\hline Inconsistent & 456 & 466 & 0.91 & 1.62 & 3.42 & 6.00 & 4.85 & 3.42 & 2.28 & 3.42 \\
\hline \multicolumn{11}{|c|}{ Small Target } \\
\hline Control & 443 & 470 & 0.82 & 4.96 & 2.00 & 3.14 & 2.57 & 4.28 & 3.71 & 1.71 \\
\hline Consistent & 450 & 482 & 6.64 & 8.26 & 4.85 & 4.28 & 4.28 & 2.00 & 3.14 & 2.57 \\
\hline Inconsistent & 441 & 491 & 8.66 & 4.55 & 3.71 & 6.28 & 4.28 & 2.57 & 2.28 & 2.28 \\
\hline
\end{tabular}

dition without context circles $[t(13)=2.569, p<.012$ for the display size of five; $t(13)=2.458, p<.015$ for the display size of seven; $t(13)=0.872, p=.200$ for the display size of three]. Combined across display size, the RT benefit for the consistent condition relative to the control condition was $10 \mathrm{msec}$.

Error analysis. Table 1 presents the error rates for all experimental conditions. An ANOVA of the error data, with the factors size of target test circle, display size, target, and size of context circles, revealed no significant main effects, but a number of interactions reached significance. Error rates - in particular, target miss errorswere, overall, higher in inconsistent conditions than in consistent conditions [target size $\times$ size of context circles, $F(2,26)=4.653, p<.032$; target size $\times$ target present/absent $\times$ size of context circles, $F(2,26)=3.901$, $p<.050]$. Furthermore, differences in error rates between consistent and inconsistent conditions increased with increasing display size [target size $\times$ display size $\times$ target present/absent $\times$ size of context circles, $F(2,26)=$ $4.687, p<.022]$. Thus, the pattern of errors confirms the RT effects.

\section{Discussion}

The results show that, in simple visual search, detection of a large target object among smaller distractor objects is independent of display size, a finding that is consistent with earlier studies (e.g., Müller et al., 1995). The flat search functions under optimal conditions confirm that object size is processed efficiently - that is, preattentively. Furthermore, there was a search asymmetry in such a way that search was more efficient with large target objects among smaller distractor objects than with small target objects among larger distractor objects. In addition, although large target objects were detected efficiently at all display sizes, search for small target objects was strongly affected by display size in the experimental conditions. This pattern of effects replicates that reported by Treisman and Gormican (1988, Experiments 1 and 1A). According to Treisman and Gormican, search asymmetries reflect the differential efficiency of search for the presence versus search for the absence of a target- defining feature. Pop-out depends on the presence of feature-based activity on a "silent" background, and search is more efficient when the target produces greater activity in the population of feature detectors relative to the background rather than vice versa.

RTs were faster when the target and distractor test circles were presented on their own (control conditions) than when they were surrounded by (inconsistent) context circles. This indicates that the presence of context objects generally interferes with search performance. The interference is likely to arise because the task-irrelevant context objects, which are similar to the test objects that are to be searched for a size difference, clutter the search display. Thus, it is reasonable to suppose that, for search to be directed to relevant (test) objects, the context objects must be suppressed to overcome their interference (which may be particularly marked under inconsistent context circle conditions).

The extent of the interference depended both on the size of the context circles and the size of the target test circle to be detected. With search for a large target test circle, RTs increased with increasing size of context circles; in contrast, with search for a small target test circle, RTs were increased with any context circles. Performance was worst overall in inconsistent conditions, when the target test circle was in between the distractor test and context circles in size. This is in line with previous studies that have shown search for in-between targets to be harder than search for targets defined by the largest or the smallest feature value in the display (e.g., Treisman \& Gelade, 1980; Wolfe, 1994, 1998). The increased search difficulty arising from this may have swamped any facilitation produced by the Ebbinghaus apparent size illusion.

However, there was evidence of facilitation with search for a large target test circle under consistent conditions (i.e., with test circles surrounded by small context circles, increasing especially the target's apparent size), for which detection RTs were expedited not only with respect to the inconsistent conditions (i.e., with test circles surrounded by large context circles, decreasing especially the distractor's apparent size), but also with respect to the control conditions (with five- and seven-item displays). 
This indicates that the apparent size illusion created by the context circles can help to overcome the general interference caused by the very same circles.

Concerning the issue of how apparent size (as opposed to retinal size) information is processed, the context circle effects obtained in Experiment 1 are revealing: Target detection is expedited in search for a large target object if the context objects affect the target's apparent size in a consistent manner-that is, if its perceived size and, thus, the target-distractor difference is magnified by small context objects. Of course, the small context objects would not only make the large target test circle but also the smaller (relative to the target) distractor test circles appear larger. However, crucially, the apparent size modulation is larger for the target than for the distractor test objects. This is the implication from Massaro and Anderson (1971), who demonstrated that the magnitude of the size contrast between test and context circles determines the magnitude of the size misjudgment in the Ebbinghaus illusion.

With search for a large target, the facilitative effect of consistent context circles was manifested not only in comparison with the inconsistent conditions but also in comparison with the control conditions, even though the mere presence of context objects can be supposed to have affected search adversely. The expedition of target detection in consistent conditions relative to control conditions was independent of display size (if anything, it was more marked with larger display sizes), indicating spatially parallel processing of the context objects. This provides evidence that apparent size information, giving rise to the Ebbinghaus illusion, is computed and represented preattentively.

However, before accepting that the Ebbinghaus apparent size illusion is causal for enhanced detectability of the large target surrounded by smaller context circles (relative to the control condition), alternative accounts must be considered. One alternative might be that performance was based on a measurement of the distance between the critical target test circle and the context circle (i.e., based on the observers applying this measurement in parallel to all configurations in the display). For a given size of context circle, the center-to-center distances were equivalent for target and distractor configurations, so that the critical distance would have had to be the perimeter-to-perimeter distance. However, this distance was equivalent for consistent and inconsistent context circle conditions (for a given blocked target circle size), and so no differential context effects should have been expected on this account; in fact, performance should have been superior to the control condition in both the consistent and inconsistent conditions, which was not the case.

Another alternative is that the observers compared all display configurations in parallel with a memory template of the large target test circle surrounded by smaller context circles (only this configuration produced faster detection RTs relative to the control condition). How- ever, such a strategy would have been nonoptimal because this configuration was presented on only one third of the target-present trials within a block. Furthermore, if the observers had applied such a strategy in the large target condition, why did they not operate a similar strategy in the small target condition (which failed to produce any context circle effects)?

One reason why the observers might have preferred the large target surrounded by smaller context circles could have been that this target configuration is a better Gestalt than are the alternative configurations. This was investigated in a follow-up study in which 20 observers were asked to judge the goodness of the various configurations. All configurations were presented simultaneously but at randomized locations on the display monitor, and observers had to rank-order the various configurations in terms of their Gestalt goodness. This study revealed no evidence of a preference by the observers for the large target with small context circles configuration. The mean rank for this configuration was 2.95 , which was equivalent to the rank for the small test with small context circles and the rank for the small test with small context circles configurations (2.90 and 2.70, respectively), whereas the large test with large context circles configuration was ranked highest (1.45). Thus, when observers searched for the large target among small distractor test circles surrounded by small context circles, there was no difference in ranked Gestalt goodness between the target and distractor configurations. Therefore, the efficient search in this condition cannot be attributed to greater Gestalt quality of the target configuration. However, when observers searched for the large target among small distractor test circles surrounded by large context circles, the Gestalt goodness was significantly greater for the target relative to the distractor configurations. Despite this difference, search performance in this condition was relatively inefficient. This pattern indicates that search performance was not determined by the subjective Gestalt goodness of the various configurations. ${ }^{3}$

In a follow-up experiment with search for a large target only (reported in Müller \& Busch, 2004), observers were presented with isoluminant but differently colored test and context circles (red and green, respectively, and vice versa) to test whether the RT modulation in the experimental conditions was caused by luminance differences between both types of circle. This experiment produced results similar to those of the present Experiment 1. That is, relative to the control condition without context circles, RTs were faster in the consistent condition (with smaller context circles) and slower in the inconsistent condition (with larger context circles). However, critically, with same-colored test and context circles, search RTs significantly increased with increasing display size, and the consistency effects were insignificant, indicating that the context circles interfered with search performance.

Thus, it would appear that the most plausible explanation for the enhanced detectability of the large target surrounded by smaller context circles is in terms of the par- 
allel coding of the Ebbinghaus apparent size illusion reinforcing the physical size differences between the target and distractor test circles.

\section{EXPERIMENT 2}

Experiment 2 was designed to further test this explanation by following an alternative approach. In an initial psychophysical experiment (Experiment 2A), the apparent sizes of the test circles in the various experimental conditions of Experiment 1 were determined, to ascertain that there were indeed differences in the test circles' apparent sizes depending on the size of the context circles. Subsequently, a visual search RT experiment (Experiment 2B) was carried out to examine whether the expedited detection of the larger target in the consistent conditions of Experiment 1 was based on a preattentive representation of the test circles' apparent size. If so, a direct correspondence was expected between the efficiency of target detection under search conditions with context circles (i.e., with effects of the Ebbinghaus illusion) and the efficiency under adapted control conditions (without context circles), in which the retinal size of the test circles matched the apparent size of the test circles (estimated in the psychophysical Experiment 2A).

\section{Experiment 2A}

\section{Method}

Participants. Twenty-four students of the Universities of Leipzig and Munich (20 female; ages ranged from 20 to 51 years) took part in Experiment 2A. The Leipzig observers participated in two experimental sessions; the Munich observers participated in only one session (performing half the number of trials of the Leipzig observers).

Stimuli and Task. Each stimulus display consisted of one Ebbinghaus configuration and one comparison circle, positioned $6 \mathrm{~cm}$ to the right and the left of the screen center and with the positions of the Ebbinghaus configuration and comparison circle varying randomly. The Ebbinghaus configuration consisted of a filled test circle either 6 or $10 \mathrm{~mm}$ in diameter, surrounded by six unfilled context circles that were 0 (i.e., no context circles), 3, 6, 10, or $16 \mathrm{~mm}$ in diameter. The size of the (filled) comparison circle varied in $0.5-\mathrm{mm}$ steps between 3 and $9 \mathrm{~mm}$ and, respectively, 7 and $13 \mathrm{~mm}$, depending on the size of the test circle. The observers were instructed to indicate which of the two filled circles appeared larger by pressing the corresponding left or right mouse button (nonspeeded response).

Design and Procedure. The independent variables were size of test circle $(6$ or $10 \mathrm{~mm})$, size of context circles $(0,3,6,10$, or $16 \mathrm{~mm}$ ), and size of the comparison circle (varying in 13 steps of $0.5 \mathrm{~mm}$ ). The observers performed the required judgments with displays of all 130 factorial combinations of these variables, presented in random order across an experimental session (10 trials per combination per session). The sessions consisted of 20 blocks of 65 trials, which took $45 \mathrm{~min}$ to complete.

\section{Results and Discussion}

Figure 3 presents the group mean frequency of the test circle larger than comparison circle response as a function of size of comparison circle, separately for the small and large test circle conditions. The size of the compar- ison circle at which the frequency of test circle larger than comparison circle responses was $50 \%$ was taken as representing the apparent size of the test circle in each condition (point of subjective equality). Table 2 summarizes the resulting apparent size estimates. As can be seen, a test circle of 6-mm diameter, for example, appeared to be $6.3 \mathrm{~mm}$ when surrounded by $3-\mathrm{mm}$ context circles, but it appeared to be $5.63 \mathrm{~mm}$ when surrounded by $16-\mathrm{mm}$ context circles. A test circle of $10-\mathrm{mm}$ diameter appeared to be $10.25 \mathrm{~mm}$ when surrounded by $3-\mathrm{mm}$ context circles, but it appeared to be $9.13 \mathrm{~mm}$ when surrounded by $16-\mathrm{mm}$ context circles. Thus, as was expected, the apparent size of the test circles varied systematically, depending on the size of the context circles: A test circle surrounded by smaller context circles appeared larger, whereas a test circle surrounded by larger context circles appeared smaller. Linear regression analyses (one-tailed tests) revealed a roughly linear dependence of the estimated Ebbinghaus illusion (i.e., a difference between apparent and retinal sizes of test circle) on the size difference between the test and context circles $\left(r^{2}=.701, p=.081\right.$ for small test circles; $r^{2}=.990, p<.002$ for large test circles). This is consistent with Massaro and Anderson's (1971) results.

\section{Experiment 2B}

\section{Method}

Participants. Sixteen students of the Universities of Leipzig and Munich (12 female; ages ranged from 21 to 36 years) took part in Experiment 2B. All the observers participated in two 45-min sessions.

Stimuli. The stimulus displays were the same as those in Experiment 1 , except for the size of the test circles in the control conditions. In the experimental conditions, the target was $10 \mathrm{~mm}$ and the distractors were $6 \mathrm{~mm}$, or the target was $6 \mathrm{~mm}$ and the distractors were $10 \mathrm{~mm}$; the context circles were $3,6,10$, or $16 \mathrm{~mm}$ (henceforth referred to as $e 3, e 6, e 10$, and $e 16$, respectively). On the basis of the estimated apparent sizes in Experiment 2A, the (retinal) sizes of the test circles in the control conditions were as follows. In the control for the 3-mm experimental condition (henceforth referred to as $c 3$ ), the test circles were 10.2 and $6.3 \mathrm{~mm}$, respectively; in the $c 6$ condition, the test circles were 10.1 and $5.8 \mathrm{~mm}$; in the $c 10$ condition, they were 9.7 and $5.8 \mathrm{~mm}$; and in the $c 16$ condition, 9.1 and $5.6 \mathrm{~mm}$.

Design and Procedure. The independent variables were size of target test circle (large or small), display size (three, five, or seven items), test condition (control or experimental), context condition $(3,6,10$, or 16 -i.e., c3, c6, c10, or c16, and e3, e6, e10, or e16, respectively), and target (present or absent).

The procedure was generally the same as that used in Experiment 1 . In one session, the target test circle was larger than the distractor test circles; in the other session, the target test circle was smaller. The order of sessions was counterbalanced across observers. The display size was kept constant throughout each trial block but varied across blocks. Context condition was varied within each block. In contrast to Experiment 1, the control and experimental conditions were blocked within each session (in Experiment 1 , control condition trials were randomly interleaved with consistent and inconsistent condition trials within the same block). Each of the two sessions consisted of 18 blocks of 74 trials (including seven or eight initial practice trials) - that is, a total of 1,200 test trials ( 25 trials per condition). 
A $\quad$ Large test circle, $10 \mathrm{~mm}$

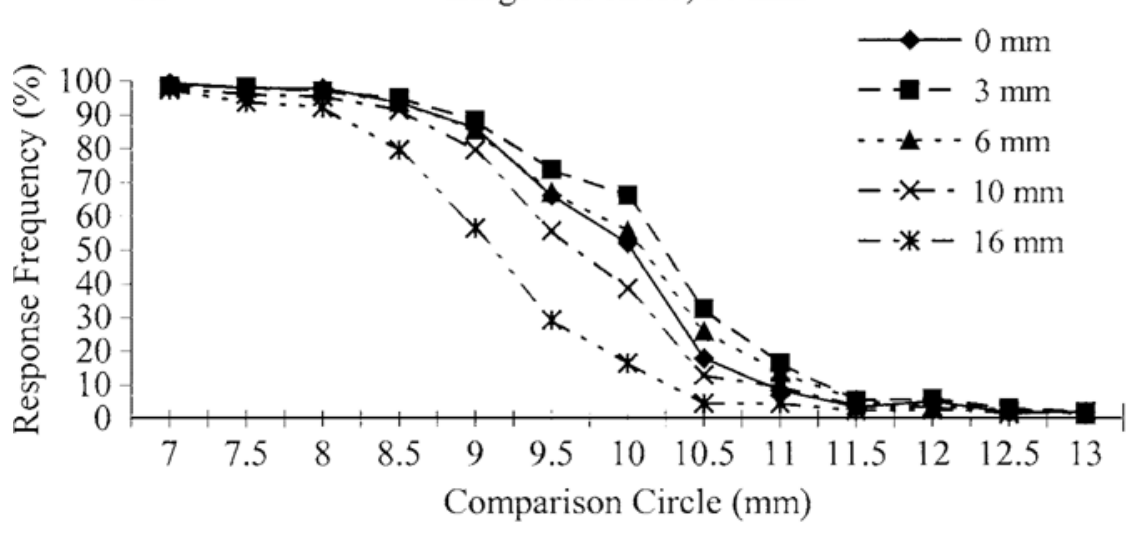

B Small test circle, $6 \mathrm{~mm}$

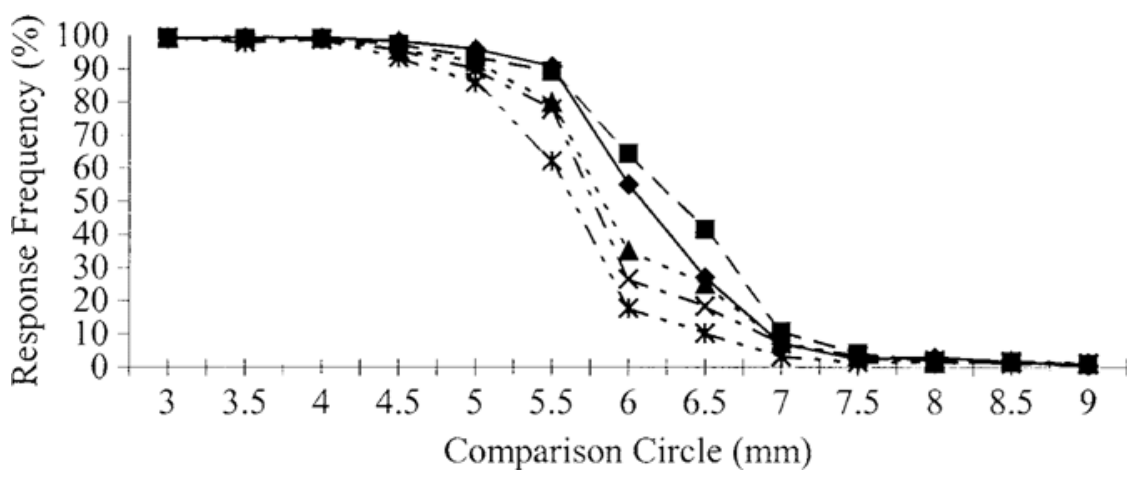

Figure 3. Mean frequency of test circle appears larger than comparison circle responses (in percentages) in Experiment 2A for (panel A) large and (panel B) small test circles. The size of context circle conditions are represented by different line types.

\section{Results}

RT analysis. Prior to analysis, outlier RTs (2.8\%) were eliminated from the data. Figure 4 presents the group mean correct target-present RTs as a function of display size for the experimental and control conditions, respectively, separately for large and small target test circles. Table 3 (left-hand side) presents summary search rate and base RT parameters. The results revealed that, with search for a large target test circle, RTs increased with increasing context in the control conditions (i.e., from $\mathrm{c} 3$ to $\mathrm{c} 16)$ and with increasing size of context circles in the experimental conditions (i.e., from e3 to e16), with a larger context (circle) effect in the experimental conditions. With search task for a small target test circle, the context condition effects were reversed but were less clear-cut (at least in the experimental conditions).

Figure 4 shows that, in both search (i.e., size of target test circle) conditions, the RT modulation caused by the varying context conditions was comparable in the experimental conditions (with context circles) with the corresponding control conditions (without context circles).

The observers' mean correct target-present RTs were examined by a repeated measures ANOVA, with size of target test circle (large or small), test condition (experimental or control), display size (three, five, or seven), and context condition $(3,6,10$, or 16$)$ as factors.

This ANOVA revealed the main effects to be (borderline) significant for all factors $[F(1,15)=4.441, p=$ .052 for size of target test circle; $F(1,15)=87.127, p<$ .001 for test condition; $F(2,30)=3.466, p=.060$ for display size; and $F(3,45)=6.306, p<.007$ for context condition]. Furthermore, the following interactions were significant: target size $\times$ test condition $[F(1,15)=11.612$, $p<.004]$, target size $\times$ display size $[F(1,15)=8.861$, $p<.003]$, and target size $\times$ context condition $[F(2,30)=$

Table 2

Apparent Sizes (in Millimeters) of the Test Circles as a Function of Size of Context Circles in Experiment 2A

\begin{tabular}{ccr}
\hline & \multicolumn{2}{c}{ Test Circles $(\mathrm{mm})$} \\
\cline { 2 - 3 } Context Circles $(\mathrm{mm})$ & 6 & 10 \\
\hline 0 & 6.09 & 10.03 \\
3 & 6.31 & 10.24 \\
6 & 5.83 & 10.09 \\
10 & 5.77 & 9.66 \\
16 & 5.64 & 9.12 \\
\hline
\end{tabular}


A Large target test circle Control conditions

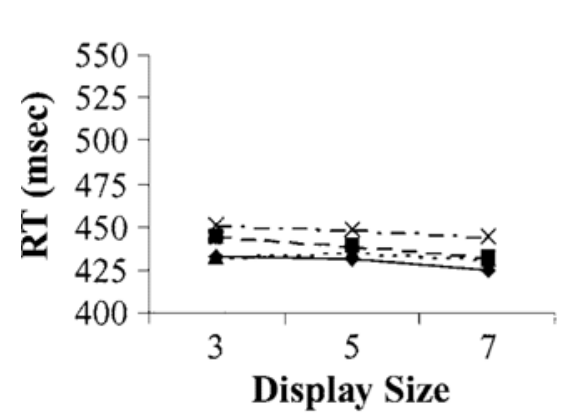

C Large target test circle Experimental conditions

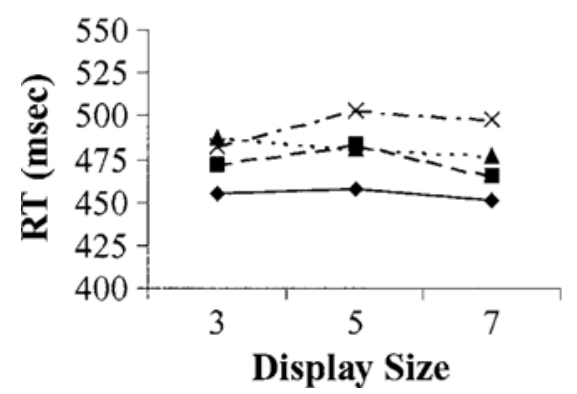

B Small target test circle Control conditions

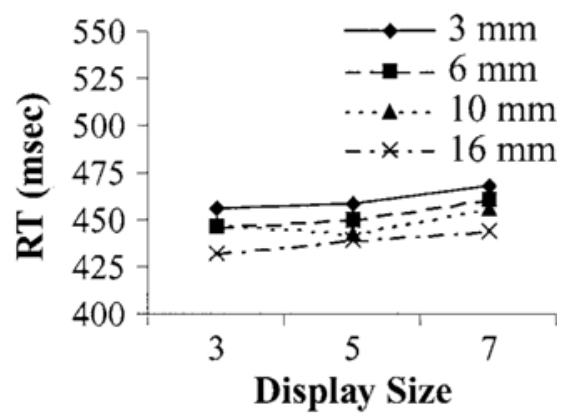

D Small target test circle Experimental conditions

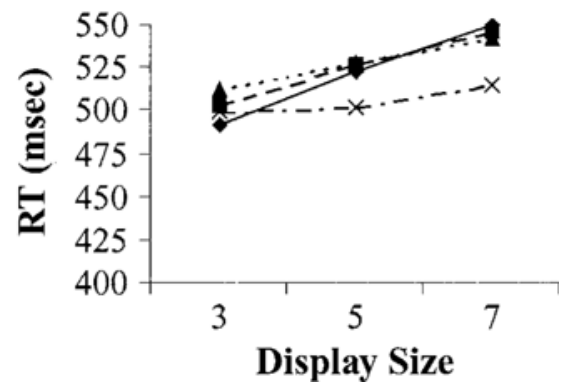

Figure 4. Mean target-present search reaction times (RTs, in milliseconds) in Experiment 2B for (panels $A$ and $C$ ) large and (panels $B$ and D) small target test circles, separately for the different context circle conditions. Panels A and B present the RTs for control conditions (without context circles), and panels $C$ and $D$ present RTs for the experimental conditions (with context circles).

29.005, $p<.001]$. RTs were faster in the control than in the experimental conditions, and somewhat faster for large than for small target test circles. However, the RT advantage for control conditions was larger for small than for large target test circles. Furthermore, RTs increased slightly with increasing display size, owing to increasing RTs for small target test circles. Finally, RTs depended on the context condition. RTs increased with increasing context (from c3 to c16) and increasing size of context circles (from e3 to e16) for large targets, but decreased (although less clearly) for small target test circles.

The interactions were examined further by two ANOVAs conducted separately for the two target size conditions (small or large), with test condition (experimental or control), display size (three, five, or seven), and context condition $(3,6,10$, or 16) as factors. For the large target condition, the ANOVA revealed significant main effects of test condition $[F(1,15)=46.975, p<.001]$ and context condition $[F(3,45)=25.592, p<.001]$, and a significant interaction between both factors $[F(3,45)=$ $9.129, p<.002]$. RTs were faster overall in control than in experimental conditions. Most important, RTs increased with increasing context in the control conditions (from c3 to c16; see Figure 4A) and with increasing size of context circles in the experimental conditions (from e3 to e16; see Figure 4C). This context (circle) effect was larger in the experimental conditions.

For the small target condition, the ANOVA revealed all main effects to be significant: test condition $[F(1,15)=$ $62.979, p<.001]$, display size $[F(2,30)=5.620, p<$ $.016]$, and size of context circles $[F(3,45)=14.720, p<$ $.001]$. However, there were no significant interactions. RTs were faster under control than under experimental conditions, increased with increasing display size, but decreased with increasing context (see Figure 4B) or with increasing size of context circles (see Figure 4D). Thus, the context (circle) effects were generally reversed relative to search for large target test circles (although less clearly so in the experimental conditions).

Error analysis. Table 3 (right-hand side) presents the error rates in Experiment 2B. An ANOVA of the error data with the factors size of target test circle, test condition, display size, target, and size of context circles revealed a number of significant effects. Target misses were more frequent than were false alarms $[4.07 \%$ vs. $2.41 \%$; target main effect, $F(1,15)=75.150, p<.001]$. More errors - in particular, target misses - were made in experimental than in control conditions $(4.64 \%$ vs. $3.49 \%$ 
Table 3

Base Reaction Times (in Milliseconds), Search Rates (in Milliseconds/Item), and Miss and False Alarm Rates (in Percentages) as a Function of Display Size, Separately for the Large and Small Target Conditions and the Test Conditions (Control, Experimental) in Experiment 2B

\begin{tabular}{|c|c|c|c|c|c|c|c|c|c|c|}
\hline \multirow{2}{*}{$\begin{array}{c}\text { Test } \\
\text { Condition }\end{array}$} & \multicolumn{2}{|c|}{ Base } & \multicolumn{2}{|c|}{ Rate } & \multicolumn{3}{|c|}{ Misses } & \multicolumn{3}{|c|}{ False Alarms } \\
\hline & Present & Absent & Present & Absent & 3 & 5 & 7 & 3 & 5 & 7 \\
\hline \multicolumn{11}{|c|}{ Large Target } \\
\hline c3 & 439 & 483 & -1.97 & -4.04 & 1.75 & 2.75 & 3.50 & 3.00 & 2.50 & 2.50 \\
\hline c6 & 453 & 472 & -2.80 & -4.17 & 3.75 & 3.75 & 5.25 & 4.25 & 3.25 & 1.25 \\
\hline $\mathrm{c} 10$ & 435 & 470 & -0.36 & -3.39 & 5.75 & 3.50 & 3.00 & 2.75 & 1.75 & 2.00 \\
\hline $\mathrm{c} 16$ & 457 & 465 & -1.69 & -3.95 & 3.75 & 3.50 & 4.00 & 1.00 & 1.50 & 0.50 \\
\hline e3 & 459 & 518 & -0.84 & -3.77 & 2.50 & 1.25 & 2.75 & 3.75 & 2.00 & 1.50 \\
\hline e6 & 482 & 501 & -1.53 & -2.81 & 4.00 & 4.00 & 2.50 & 1.00 & 2.50 & 1.75 \\
\hline $\mathrm{e} 10$ & 496 & 515 & -2.79 & -2.59 & 4.25 & 5.00 & 3.25 & 3.25 & 2.75 & 3.75 \\
\hline $\mathrm{e} 16$ & 475 & 514 & 3.81 & -0.53 & 5.00 & 5.25 & 5.00 & 1.00 & 2.75 & 3.00 \\
\hline \multicolumn{11}{|c|}{ Small Target } \\
\hline c3 & 445 & 459 & 3.11 & -1.00 & 3.50 & 3.75 & 5.00 & 3.25 & 1.00 & 1.75 \\
\hline c6 & 434 & 461 & 3.55 & 0.78 & 4.75 & 3.00 & 3.75 & 2.00 & 1.00 & 1.25 \\
\hline $\mathrm{c} 10$ & 438 & 462 & 2.14 & 0.33 & 2.75 & 2.75 & 2.00 & 4.25 & 3.50 & 1.25 \\
\hline $\mathrm{c} 16$ & 425 & 479 & 2.77 & 1.08 & 2.50 & 3.25 & 2.50 & 5.25 & 4.25 & 3.00 \\
\hline e3 & 449 & 509 & 14.45 & 1.56 & 4.50 & 6.00 & 11.00 & 2.25 & 2.25 & 1.50 \\
\hline e6 & 473 & 460 & 10.47 & 13.94 & 3.75 & 6.75 & 5.50 & 3.00 & 2.50 & 1.00 \\
\hline $\mathrm{e} 10$ & 490 & 432 & 7.47 & 18.09 & 6.50 & 6.25 & 8.50 & 2.00 & 1.75 & 0.75 \\
\hline e16 & 484 & 456 & 4.12 & 20.98 & 2.50 & 2.75 & 2.50 & 4.00 & 3.75 & 1.50 \\
\hline
\end{tabular}

misses, $2.31 \%$ vs. $2.42 \%$ false alarms; test condition main effect, $F(1,15)=8.260, p<.012$; test condition $\times$ target interaction, $F(1,15)=11.613, p<.004]$. Although target misses tended to increase with display size, false alarms tended to decrease [display size $\times$ target, $F(2,30)=$ $8.396, p<.004]$. Both the test condition and the display size effects were more marked with small target test circles [5.54\% vs. 3.29\% misses, experimental vs. control, for the small targets; $3.73 \%$ vs. $3.69 \%$ misses, experimental vs. control, for the large targets; size of target test circle $\times$ test condition $\times$ target, $F(1,15)=11.630, p<.004$; size of target test circle $\times$ target $\times$ display size, $F(2,30)=7.241$, $p<.007$; size of target test circle $\times$ test condition $\times$ target $\times$ display size, $F(2,30)=5.062, p<.022]$.

With large target test circles, error (miss) rates were higher when test circles were surrounded by large context circles; in contrast, with small target test circles, error (miss) rates were higher when test circles were surrounded by small context circles [size of target test circle $\times$ size of context circles, $F(3,45)=4.156, p<.029$; size of target test circle $\times$ size of context circles $\times$ target, $F(3,45)=9.381, p<.001]$.

For small targets, this (miss rate) effect was also dependent on the size of the context circles and the display size, particularly under experimental conditions [test condition $\times$ size of context circles $\times$ display size, $F(6,90)=$ $4.120, p<.024$; size of target test circle $\times$ size of context circles $\times$ test condition, $F(3,45)=4.657, p<.020$; size of target test circle $\times$ size of context circles $\times$ target $\times$ test condition, $F(3,45)=3.582, p<.044$; fiveway interaction, $F(6,90)=3.644, p<.035]$.

Thus, overall, the error and, in particular, miss rate effects were in agreement with the RT effects.

\section{Discussion}

Detection of an apparently larger target was independent of display size, whereas detection of a smaller target was dependent on display size. This pattern is consistent with the pattern of results in Experiment 1.

Furthermore, detection RTs were slower overall in the experimental relative to the control conditions, although no difference had been expected. The RT slowing even applied to the large target consistent condition (10-mm target test circle, 3-mm context circles). Recall that, in Experiment 1, detection RTs were significantly faster under the large target consistent condition relative to the unadjusted control condition.

However, although detection RTs were slower, overall, in the experimental than in the control conditions, importantly, both conditions exhibited corresponding patterns of effects. For the large target conditions, RTs increased with increasing size of context circles in the experimental conditions as well as the corresponding control conditions; in contrast, for the small target conditions, RTs decreased with increasing size of context circles in the experimental conditions as well as the corresponding control conditions. This pattern of effects suggests that, in the experimental conditions, visual search was modulated by the apparent size of the test circles, in correspondence with the adjusted target and distractor circle sizes in the control conditions.

Yet RTs were slower, overall, in the experimental conditions than in the control conditions by some 40 and $70 \mathrm{msec}$ for the large and small target conditions, respectively. This general slowing of RTs may be attributable, at least in part, to the fact that, in Experiment 2B, experimental conditions (with context circles) and con- 
trol conditions (without context circles) were presented in separate trial blocks (in contrast, in Experiment 1, experimental and control conditions were presented in random order within blocks) and that no context circles were presented on half of the trials (in contrast, in Experiment 1 , only one third of the trials was without context circles). Thus, the blocked (context circle) design of Experiment 2B may have encouraged the observers to adopt a strategy different from that in Experiment 1. In particular, in Experiment 2B, the observers would not have attempted to suppress context circles on control condition trials, because there were never any context circles in (blocked) control conditions. In contrast, in Experiment 1, the observers may have used a suppression strategy on all-experimental and control-trials, since experimental trials were more frequent (two thirds as compared with one third) and since control trials were randomly interspersed with experimental trials. Thus, the overall slower RTs in the experimental conditions of Experiment $2 \mathrm{~B}$ may reflect additional processes of suppressing the (task-irrelevant) context circles that act as additional distractors under these conditions.

Context circle suppression would also explain why, with large target test circles, the differences in results among the sizes of context circles were greater for experimental (with context circles) than for control (without context circles) conditions. A possible cause of this effect is that, with search for a large target test circle, larger context circles require increased suppression.

\section{EXPERIMENT 3}

One problem with this account remains. Similar to the results of earlier studies (e.g., Aks \& Enns, 1996; Found \& Müller, 2001; Humphreys et al., 1994), the evidence for parallel coding of the Ebbinghaus apparent size illusion provided by Experiments 1 and 2 rests on base RT (intercept) rather than search rate (slope) effects. (With search for large targets, slope effects were not expected, given that the search rates were near $0 \mathrm{msec} /$ item under control conditions - see Tables 1 and 3 - and, thus, could not be improved by the operation of the Ebbinghaus apparent size illusion.) The intercept effects leave the possibility that the observers detected the target test circle on the basis of its retinal size difference relative to the size of the distractor test circles and that they were deriving apparent size information and modulating their RTs only after having deployed focal attention to the target. Thus, Experiments 1 and 2 do not conclusively answer whether apparent size is computed separately for each Ebbinghaus configuration in the display.

To address this issue, in Experiment 3, the observers were presented with heterogeneous (rather than homogeneous) displays, each of them containing the following in equal numbers: test circles surrounded by small context circles, test circles surrounded by large context circles, and test circles without context circles. Any of the three types of test circle was equally likely to be the target ob- ject on a given trial. The heterogeneous context circles were intended to make it harder for the observers to base their search solely on retinal size comparisons of the test circles (because it would be harder to direct suppression to heterogeneous context circles). If, nevertheless, the same pattern of results would be obtained as that in (the homogeneous context circle conditions of) Experiment 1, the case would be strengthened for the suggestion that apparent size is computed separately for each test circle.

\section{Method}

Participants. Twelve students of the Ludwig-MaximiliansUniversität München (10 female; ages ranged from 19 to 30 years) took part in Experiment 3.

Stimuli. The stimulus displays were similar to those used in Experiment 1, except that the displays (of three, six, or nine test circles) were heterogeneous, each containing, in equal numbers, (one, two, or three) test circles with small context circles, large context circles, and no context circles (see Figure 5 for an example display). To avoid overlapping of the various configurations, the radius of the imaginary circle around which the configurations were arranged was increased to $100 \mathrm{~mm}$ (as compared with $70 \mathrm{~mm}$ in Experiment 1).

Design and Procedure. The independent variables were size of target test circle (small or large), display size (three, six, or nine), size of context circles surrounding the target test circle (zero, small, or large), and target (present or absent).

The procedure was the same as that in Experiment 1. The experimental session was divided into two halves, each devoted to one of two search conditions: target test circle smaller than the distractor test circles and target test circle larger than the distractor test circles. The observers performed the two conditions in counterbalanced order. The display size was kept constant within each trial block, with order of blocks also counterbalanced across observers. The session consisted of 18 blocks of 60 test trials (plus 6 unrecorded practice trials) overall and lasted approximately $50 \mathrm{~min}$.

\section{Results}

RT analysis. Prior to analysis, outlier RTs (2.1\%) were eliminated from the data. Figure 6 presents the group mean correct target-present RTs as a function of display size for the small and large target conditions, respectively. Table 4 presents a summary of search rates and base RTs.

As can be seen from Figure 6, with heterogeneous search displays, the pattern of target-present RTs was
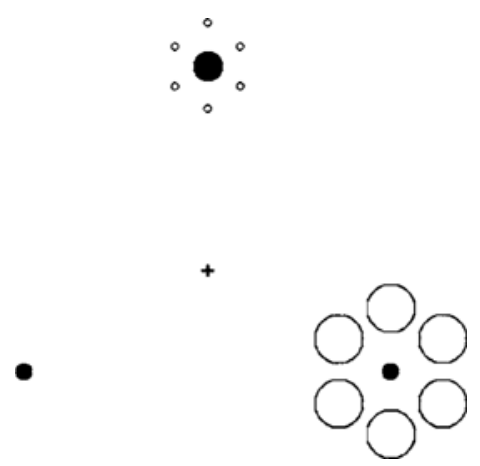

Figure 5. Example of a display for the consistent search condition for the large target condition in Experiment 3. 
A Large target test circle Target present

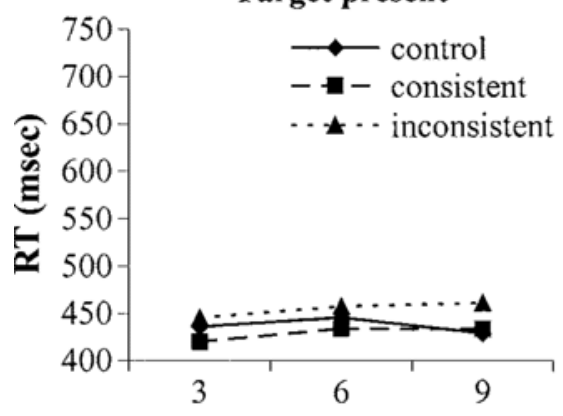

Display Size
B Small target test circle Target present

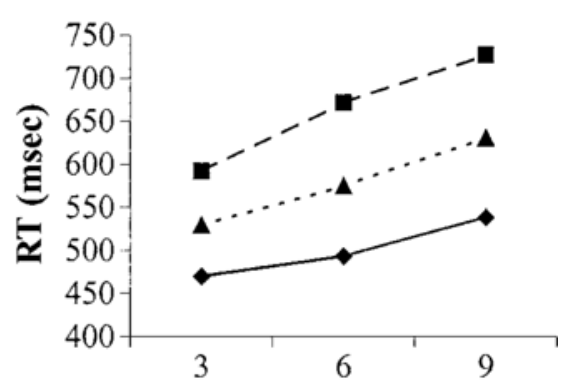

Display Size

Figure 6. Mean target-present search reaction times (RTs, in milliseconds) in Experiment 3 for (panel A) large and (panel B) small target test circles. Control, consistent, and inconsistent conditions are represented by solid, broken, and dotted lines, respectively.

very similar to that observed with homogeneous search displays (Experiment 1; see Figure 2). In particular, in the large target condition, RTs were expedited, relative to the control condition, under consistent conditions (small context circles) and slowed under inconsistent conditions (large context circles).

The observers' mean correct target-present RTs were examined by a repeated measures ANOVA, with target size (small or large), display size (three, six, or nine), and size of context circles surrounding the target test circle (zero, small, or large) as factors. All main effects were significant [target size, $F(1,11)=23.863, p<.001$; display size, $F(2,22)=8.573, p<.007$; and size of context circles, $F(2,22)=23.500, p<.001]$. Furthermore, the following interactions were significant: target $\times$ display size $[F(2,22)=12.517, p<.002]$, target $\times$ size of context circles $[F(2,22)=30.794, p<.001]$, display size $\times$ size of context circles $[F(4,44)=5.397, p<.021]$, and the three-way interaction $[F(4,44)=0.695$, n.s.]. RTs were faster when the observers searched for a large, as compared with a small, target test circle. RTs increased with increasing display size, largely owing to the in- crease when observers searched for a small target. And RTs increased overall with increasing size of the context circles. However, the effect of the size of context circles was dependent on the target size condition (target size $\times$ size of context circles interaction). This dependency was explored further by separate ANOVAs for small and large target conditions, each with display size and size of context circles as factors.

For the large target condition, only size of context circles significantly influenced RTs $[F(2,22)=15.068$, $p<.001$; display size, $F(2,22)=1.292$; display size $\times$ size of context circles, $F(4,44)=0.697]$. RTs were fastest to target test circles surrounded by smaller context circles $(436 \mathrm{msec})$, intermediate to targets without context circles (428 $\mathrm{msec})$, and slowest to targets surrounded by larger context circles (454 msec). This pattern mirrors that observed in Experiment 1.

For the small target condition, the ANOVA revealed significant effects of display size $[F(2,22)=13.874, p<$ $.001]$ and size of context circles $[F(2,22)=26.723, p<$ $.001]$, and a significant display size $\times$ size of context circles interaction $[F(4,44)=4.445, p<.035]$. RTs in-

Table 4

Base Reaction Times (in Milliseconds), Search Rates (in Milliseconds/Item), and Miss and False Alarm Rates (in Percentages) as a Function of Display Size, Separately for the Large and Small Target Conditions and the Context Conditions (Control, Consistent, Inconsistent) in Experiment 3

\begin{tabular}{|c|c|c|c|c|c|c|c|c|c|c|}
\hline \multirow{2}{*}{$\begin{array}{c}\text { Context } \\
\text { Condition }\end{array}$} & \multicolumn{2}{|c|}{ Base } & \multicolumn{2}{|c|}{ Rate } & \multicolumn{3}{|c|}{ Misses } & \multicolumn{3}{|c|}{ False Alarms } \\
\hline & Present & Absent & Present & Absent & 3 & 6 & 9 & 3 & 6 & 9 \\
\hline \multicolumn{11}{|c|}{ Large Target } \\
\hline Control & 443 & 477 & -1.17 & 1.50 & 3.34 & 2.51 & 4.17 & 2.88 & 1.86 & 2.05 \\
\hline Consistent & 414 & & 2.33 & & 3.62 & 4.17 & 3.62 & & & \\
\hline Inconsistent & 439 & & 2.50 & & 4.73 & 3.90 & 4.73 & & & \\
\hline \multicolumn{11}{|c|}{ Small Target } \\
\hline Control & 379 & 512 & 20.17 & 24.67 & 3.62 & 2.23 & 1.67 & 3.53 & 2.32 & 3.79 \\
\hline Consistent & 591 & & 6.50 & & 10.01 & 19.73 & 21.95 & & & \\
\hline Inconsistent & 531 & & 1.50 & & 5.01 & 4.17 & 11.40 & & & \\
\hline
\end{tabular}


creased with increasing display size and with increasing size of context circles $(500,578$, and $631 \mathrm{msec}$ for zero, small, and large context circles, respectively). The context circle effect was larger for larger display sizes.

Error analysis. Table 4 presents the error rates. Overall, the observers produced $6.37 \%$ target miss errors (targetpresent trials) and $2.57 \%$ false alarm errors (target-absent trials). The error data were examined by separate ANOVAs of target misses (target size $\times$ display size $\times$ size of context circles) and false alarms (target size $\times$ display size).

Target miss errors were more frequent when the observers searched for a small target $[F(1,11)=14.822$, $p<.003]$. They increased with increasing display size $[F(2,22)=7.835, p<.009]$ and with increasing size of context circles $[F(2,22)=10.543, p<.003]$. Both the display size effect and the context circle effect was larger for the small than for the large target condition [target size $\times$ display size, $F(2,22)=9.526, p<.005$; target size $\times$ size of context circles, $F(2,22)=10.585, p<.003$; target size $\times$ display size $\times$ size of context circles, $F(4,44)=$ $4.860, p<.028]$. The ANOVA of the false alarm data revealed no significant effects.

\section{Discussion}

The results of Experiment 3 mirror those of Experiment 1 . This is especially true with search for large targets, in which case target test circles surrounded by smaller context circles were detected more quickly (consistent conditions) and target test circles surrounded by larger context circles more slowly (inconsistent conditions) than were target test circles without context circles, independent of display size. Importantly, in Experiment 3, this pattern was obtained under conditions in which at least one instance of each type of test circle was represented in the display and had an equal chance of being a (larger) target. This strongly suggests that the apparent size modulation worked separately for each single Ebbinghaus configuration in the display.

One objection that may be leveled against this interpretation is that the additive RT effects between the various context circle conditions reflect serial processing of three types of display elements: Configurations with small context circles are (for some reason) searched first for the presence of a target, then elements without context circles are searched next, and finally configurations with large context circles are searched. Since the different types of elements are searched and compared in parallel, the result is an additive RT effect of context circle condition (that is independent of display size). However, this account fails to explain why the effect was manifest with three-element displays (that contained one element of each type), in which case target presence could be ascertained only by comparisons across the various types of configuration. Second, it fails to explain why elements with large context circles would have a lower priority of processing than elements without context circles or elements with small context circles. An account in terms of saliency fails because, arguably, large context circle configurations are more salient than are test circles without or with small context circles. One possibility that remains is that configurations with large context circles were processed last because the test circles appeared smallest (and configurations with small context circles first because the test circles appeared largest). However, if this were true, the account would have to admit a role of the Ebbinghaus apparent size modulation in (preattentively) determining the order with which the various types of display elements were processed. Thus, in summary, it seems difficult to account for the data of Experiment 3 without assuming that the apparent size information is derived separately (i.e., spatially in parallel) for all Ebbinghaus configurations in the search display.

\section{EXPERIMENT 4}

Experiment 4 was carried out to examine whether the influence of the context circles on the test circles in the Ebbinghaus illusion, as was demonstrated in Experiments 1 and 2B, would also be manifested in a more difficult search task. If the effects of the context circles make the target test circle appear to be substantially larger or smaller (depending on whether observers have to search for a large or a small target test circle), then target detection might be successful even if the target and distractor test circles are less easily discriminable. To test this, the size difference between target and distractor test circles was reduced in Experiment 4. If observers can make use of the misjudgment effect of the Ebbinghaus illusion, one would expect a facilitative effect at least in the consistent conditions of this difficult search task (i.e., with a large target test circle surrounded by small context circles and with a small target test circle surrounded by large context circles), because the apparent size illusion provides a source of information, making the task solvable.

\section{Method}

Participants. Eighteen students of the University of Leipzig (14 female; ages ranged from 19 to 47 years) took part in two experimental sessions.

Stimuli. The stimulus displays were the same as those in Experiment 1 , except for the size of the test circles. A preliminary experiment, with fixed target $(10 \mathrm{~mm})$ and variable distractor sizes across trials (without context circles), was carried out to determine the minimum size difference between the target and distractor test circles required to discriminate between them reliably. When the target and distractor test circles differed in diameter by at least $3 \mathrm{~mm}$, the presence of a smaller or larger target test circle was well detectable. When they differed by only $1 \mathrm{~mm}$, they were no longer discriminable above chance. On the basis of this information, the test circles introduced in Experiment 4 were either 9 or $10 \mathrm{~mm}$ in diameter (i.e., 1-mm difference between target and distractors), and the context circles were either 3 or $16 \mathrm{~mm}$. The size of the context circles was kept constant throughout each trial block.

Design and Procedure. The independent variables were size of target test circle (small or large), display size (three, five, or seven), size of context circles (zero, small, or large), and target (present or absent).

The procedure was the same as that used in Experiment 1, except that all the observers participated in two sessions: one requiring 
A Large target test circle Target present

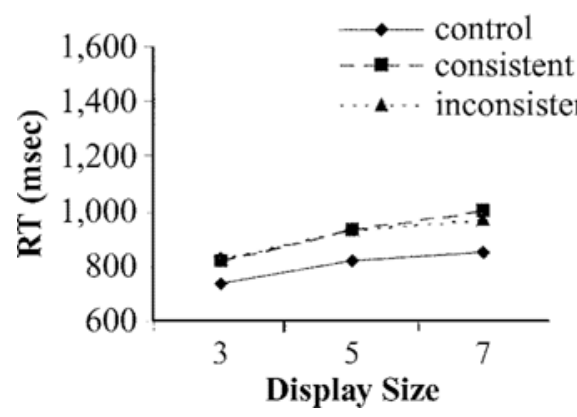

B Small target test circle

Target present

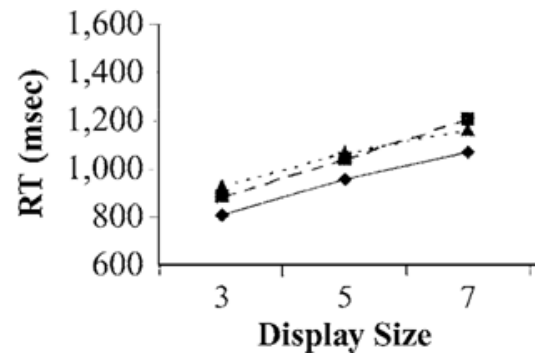

Figure 7. Mean target-present search reaction times (RTs, in milliseconds) in Experiment 4 for (panel A) large and (panel B) small target test circles. Control, consistent, and inconsistent conditions are represented by solid, broken, and dotted lines, respectively.

search for a large target test circle (10-mm target, 9-mm distractors) and the other search for a small target test circle (9-mm target, 10 -mm distractors). The order of target sessions was counterbalanced across the 18 observers. Each session consisted of 18 blocks of 40 trials, with each block consisting of 15 practice and 25 test trials (totaling 900 test trials). The observers were provided with 15 initial practice trials to help them reduce the high error rates they displayed at the beginning of a trial block. Taken together, both sessions lasted $90 \mathrm{~min}$.

\section{Results}

RT analysis. Prior to analysis, outlier RTs (5.0\%) were eliminated from the data. Figure 7 presents the group mean correct target-present RTs as a function of display size for the small and large target (test circle) conditions, respectively. Table 5 presents summary search rate and base RT statistics.

As can be seen from Figure 7, when the target test circle was hard to discriminate from the distractor test circles, there were no facilitative effects of (consistent) context circles in comparison with the control condition, not even with large targets, for which consistent (i.e., smaller) context circles had expedited detection when the target and distractor test circles were easy to discriminate (see Figures 2 and 6).
The observers' mean correct target-present RTs were examined by a repeated measures ANOVA, with target size (small or large), display size (three, five, or seven), and size of context circles (zero, small, or large) as factors.

The ANOVA revealed all main effects to be significant [target size, $F(1,17)=9.944, p<.006$; display size, $F(2,34)=22.488, p<.001$; size of context circles, $F(2,34)=16.171, p<.001]$. As in Experiment 1, RTs were slower when the observers searched for a small target test circle than when they searched for a large one (i.e., search asymmetry). Furthermore, RTs increased with increasing display size for both large and small target test circles. And RTs increased when there were context circles in the display, relative to the control conditions (which themselves showed a display size dependent RT increase). Display size exerted a stronger effect on target detection when the target test circle was small rather than large [ 68 vs. 36 msec per item; target size $X$ display size interaction, $F(2,34)=6.360, p<.009]$. Importantly, there was no evidence for differential effects between the consistent and inconsistent context circle conditions [the target size $\times$ size of context circles interaction was not significant, $F(2,34)=0.061$, and planned comparisons between the consistent and inconsistent

Table 5

Base Reaction Times (in Milliseconds), Search Rates (in Milliseconds/Item), and Miss and False Alarm Rates (in Percentages) as a Function of Display Size, Separately for the Large and Small Target Conditions and the Context Conditions (Control, Consistent, Inconsistent) in Experiment 4

\begin{tabular}{|c|c|c|c|c|c|c|c|c|c|c|}
\hline \multirow{2}{*}{$\begin{array}{c}\text { Context } \\
\text { Condition } \\
\end{array}$} & \multicolumn{2}{|c|}{ Base } & \multicolumn{2}{|c|}{ Rate } & \multicolumn{3}{|c|}{ Misses } & \multicolumn{3}{|c|}{ False Alarms } \\
\hline & Present & Absent & Present & Absent & 3 & 5 & 7 & 3 & 5 & 7 \\
\hline \multicolumn{11}{|c|}{ Large Target } \\
\hline Control & 662 & 687 & 28.36 & 54.15 & 10.88 & 13.11 & 17.11 & 6.44 & 7.77 & 6.00 \\
\hline Consistent & 694 & 738 & 45.15 & 79.40 & 17.33 & 15.77 & 23.33 & 8.00 & 10.66 & 8.22 \\
\hline Inconsistent & 729 & 652 & 36.79 & 91.71 & 10.44 & 14.66 & 18.22 & 4.22 & 6.00 & 5.55 \\
\hline \multicolumn{11}{|c|}{ Small Target } \\
\hline Control & 619 & 589 & 65.00 & 126.82 & 15.77 & 16.66 & 16.88 & 7.77 & 7.77 & 7.77 \\
\hline Consistent & 650 & 730 & 79.40 & 114.51 & 10.66 & 14.22 & 17.55 & 9.55 & 7.77 & 7.11 \\
\hline Inconsistent & 751 & 715 & 60.62 & 116.83 & 17.77 & 20.00 & 28.66 & 11.55 & 12.00 & 9.77 \\
\hline
\end{tabular}


search conditions showed no significant RT differences for either target size condition. Thus, there was no evidence of facilitation of target detection by the presentation of consistent context circles in comparison with inconsistent circles.

Error analysis. Table 5 presents the error rates in Experiment 4 . The error data were examined by an ANOVA with the factors size of target test circle, display size, target, and size of context circles. Target miss errors were more frequent than were false alarms [target, $F(1,17)=$ $62.475, p<.001]$, more errors were made with large context circles [size of context circles, $F(2,34)=13.192$, $p<.001]$, and error rates - in particular, target miss rates - increased with increasing display size [display size, $F(2,34)=4.497, p<.028$; display size $\times$ target interaction, $F(2,34)=5.555, p<.015]$. This increase was larger when context circles were smaller than the test circles were [display size $\times$ target $\times$ size of context circles, $F(4,68)=5.948, p<.005]$, and it tended to be larger when the target test circles were smaller than the distractor test circles were [size of target test circle $\times$ display size $\times$ target $\times$ size of context circles, $F(4,68)=$ $3.080, p<.052]$.

\section{Discussion}

The results of Experiment 4 are consistent with reports (e.g., Treisman \& Gormican, 1988) that steep RT slopes result in (feature) search tasks in which the target and distractor objects are difficult to discriminate (see also Duncan \& Humphreys, 1989), perhaps because the display needs to be scanned serially. The search functions for target-present and -absent RTs show the 1:2 slope ratio considered to be characteristic of serial, selfterminating search. The slope ratio for search for a small target was 1.00:1.75 (68:119 msec), and that for search for a large target was 1.00:2.08 (36:75 msec). Moreover, like the observers in Experiment 1, the observers in Experiment 4 took significantly longer to detect a small target object in a display of larger distractor objects than vice versa, providing further evidence of a search asymmetry for size-defined targets (see Treisman \& Gormican, 1988). Interestingly, this asymmetry was evident despite the task's being hard overall (producing search RTs that increased significantly with the number of Ebbinghaus configurations in the display). In fact, the asymmetry was more marked than that in Experiment 1 (in which the task was easy, producing search RTs independent of display size), and it increased with increasing display size (the search RT slopes were approximately twice as steep with small targets as with large targets; 68 vs. $36 \mathrm{msec}$ per item for target-present responses).

The aim of Experiment 4 was to assess the extent of the misjudgment influence of the context circles on the test circles under hard search conditions. Even under such conditions, consistent context circles were expected to make the target test circle appear larger (or smaller) in the appropriate search conditions. Since the size contrast between the target test and context circles was still larger than was the contrast between the distractor test and context circles, the consistent context circles would have influenced the apparent size of the target test circle more than that of the distractor test circles. However, the results suggest that the apparent size illusion was not beneficial to search performance, not even under consistent conditions. Instead, the presence of context circles in the display was detrimental to search performance overall. One reason why the original hypothesis was disconfirmed may be that, when target detection is hard, observers tend to suppress the (task-irrelevant and potentially distracting) context circles (see below).

Judging from the error rates, the observers were able to detect target objects reasonably well, despite the difficult target-distractor discrimination. (The finding that the observers were able to perform the search with an accuracy well above chance in Experiment 4 may have been caused by the fact that distractor size was fixed within trial blocks; in the pilot experiment to Experiment 4 , it was variable.) But the RTs were slowed when context circles were added to the displays - in the control conditions, the observers responded more rapidly (and their search rates were faster). In other words, the addition of context circles to the search displays produced general interference, so much so that apparent size modulation effects were completely swamped by it.

One possible reason why there may have been no apparent size modulation effects is that the observers scanned the display configurations serially (i.e., without being able to compare the target and distractor test circles in parallel). In this case, the observers would have had to establish and maintain a memory representation, or template (Duncan \& Humphreys, 1989), of the target size with which to compare a given scanned display item. Apparent size modulation effects would not be expected under these conditions, because the template might include a representation of the context circles (in addition to the target test circle) within a given block (recall that the size of the context circles was blocked in Experiment 4). It may also be that observers scan one item, maintain its size in memory, and then scan the next item to ascertain whether it is different in size from the memorized item. Perhaps, to optimize such comparisons, observers must suppress the task-irrelevant context circles (i.e., not represent them in memory), since including a representation of the context circles potentially could be confusing. That memory processes along one or the other line played a role in Experiment 4 may be inferred from the high rate of errors, indicating processes of matching the internal template of the target against the display stimuli. These processes produce a high rate of errors, false alarms as well as target misses, because the template has to be updated frequently.

The Ebbinghaus illusion was not strong enough to produce any facilitative effects in Experiment 4 . If the context circles had been suppressed, there would have been no apparent size modulation effects in the various context-circle conditions. Thus, the prolonged RTs under 
consistent and inconsistent conditions in Experiment 4 may reflect costs associated with suppressing the taskirrelevant context circles, which are similar under both conditions. The suppression may be more difficult under hard (Experiment 4) than under standard (Experiment 1) search conditions, swamping any differential apparent size effects.

\section{EXPERIMENT 5}

Experiment 5 was designed to test whether, under efficient search conditions, observers do indeed suppress the (task-irrelevant) context circles in order to more effectively direct comparisons to the (task-relevant) target and distractor test circles, without interference from the context circles. To test this, observers were provided with a 750msec preview of the context circles before the presentation of the target and distractor test circles. If context circles interfered with search (under no-preview conditions), it was expected that the preview would provide observers with the opportunity to suppress the context circles in advance of test circle onset. Consequently, the preview would lead to the abolishing of any apparent size modulation effects produced by the context circles under nopreview conditions (Experiment 1). The underlying idea was that context circle suppression operates along lines similar to the visual-marking processes recently demonstrated by Watson and Humphreys (1997) - that is, in terms of a parallel top-down process of marking off (inhibiting) items that do not fit with the target description (template; see also Egeth, Virzi, \& Garbart, 1984; Kaptein, Theeuwes, \& van der Heijden, 1995). There is evidence in support of this idea from psychophysical studies, in which the Ebbinghaus apparent size illusion was significantly reduced when the context circles were presented in advance of the test circle (Cooper \& Weintraub, 1970; Jaeger \& Pollack, 1977). Note, however, that these studies presented only one Ebbinghaus configuration at a time.

\section{Method}

Participants. Twelve students of the University of Leipzig (10 female; ages ranged from 19 to 29 years) took part in a single experimental session.

Stimuli. The stimuli were identical to those in Experiment 1, except for the successive presentation of the context and test circles (see Design and Procedure section).

Design and Procedure. The independent variables were size of target test circle (small or large), display size (three, five, or seven), target (present or absent), and size of context circles (zero, small, or large). The experimental session consisted of nine blocks with small and nine with large target test circles (with 10 practice and 50 test trials per block for a total of 900 trials) and lasted approximately $45 \mathrm{~min}$.

The procedure was identical to that in Experiment 1, except that the context circles were presented earlier than the test circles were. Each trial began with the presentation, for $750 \mathrm{msec}$, of the black fixation cross in the center of the screen and the context circles arranged around the positions of the (subsequently presented) test circles. Next, the test circles were displayed (in addition to the context circles and fixation marker). The Ebbinghaus configurations then remained in view until a response was made. In the control conditions, only the central fixation cross was presented for the period of $750 \mathrm{msec}$, at the end of which the onset of the target and distractor test circles without any context circles occurred. The instruction was the same as in Experiment 1 (i.e., the observers were not told to try to ignore or suppress the context circles).

\section{Results}

RT analysis. Prior to analysis, outlier RTs (5.4\%) were eliminated from the data. Figure 8 presents the group mean RTs as a function of display size for the small and large target (test circle) conditions, respectively. Table 6 presents summary search rate and base RT statistics.

As can be seen from Figure 8, with a preview of the context circles, all facilitative effects of the context circles were diminished (small target condition) or disappeared entirely (large target condition).

The observers' mean correct target-present RTs were examined by a repeated measures ANOVA, with size of target test circles (small or large), display size (three, five, or seven), and size of context circles (zero, small,

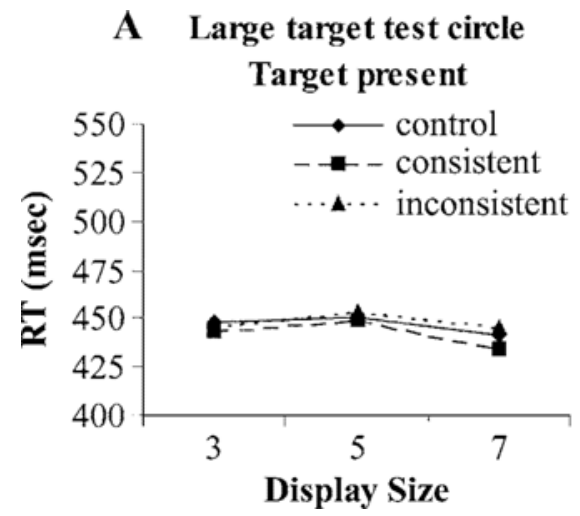

\section{B Small target test circle Target present}

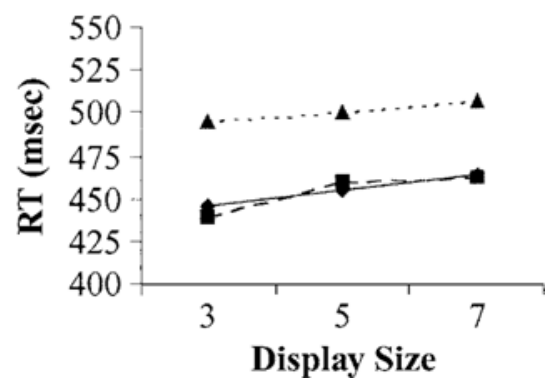

Figure 8. Mean target-present search reaction times (RTs, in milliseconds) in Experiment 5 for (panel A) large and (panel B) small target test circles. Control, consistent, and inconsistent conditions are represented by solid, broken, and dotted lines, respectively. 
Table 6

Base Reaction Time (in Milliseconds), Search Rate (in Milliseconds/Item), and Miss and False Alarm Rates (in Percent) as a Function of Display Size, Separately for the Large and Small-Target Conditions and the Context Conditions (Control, Consistent, Inconsistent) in Experiment 5

\begin{tabular}{|c|c|c|c|c|c|c|c|c|c|c|}
\hline \multirow{2}{*}{$\begin{array}{c}\text { Context } \\
\text { Condition } \\
\end{array}$} & \multicolumn{2}{|c|}{ Base } & \multicolumn{2}{|c|}{ Rate } & \multicolumn{3}{|c|}{ Misses } & \multicolumn{3}{|c|}{ False Alarms } \\
\hline & Present & Absent & Present & Absent & 3 & 5 & 7 & 3 & 5 & 7 \\
\hline \multicolumn{11}{|c|}{ Large Target } \\
\hline Control & 454 & 455 & -1.68 & 1.58 & 1.66 & 1.33 & 2.00 & 3.00 & 1.66 & 0.66 \\
\hline Consistent & 454 & 468 & -2.45 & 3.54 & 2.33 & 2.33 & 2.66 & 4.66 & 3.00 & 3.00 \\
\hline Inconsistent & 449 & 461 & -0.12 & 0.41 & 1.66 & 3.00 & 3.66 & 0.66 & 2.00 & 1.33 \\
\hline \multicolumn{11}{|c|}{ Small Target } \\
\hline Control & 432 & 462 & 4.48 & 6.27 & 2.00 & 2.66 & 1.33 & 3.33 & 2.66 & 3.33 \\
\hline Consistent & 424 & 476 & 5.98 & 5.66 & 2.66 & 1.66 & 2.66 & 2.66 & 2.33 & 2.66 \\
\hline Inconsistent & 485 & 474 & 3.12 & 2.97 & 4.66 & 4.33 & 5.00 & 2.00 & 1.00 & 1.00 \\
\hline
\end{tabular}

or large) as factors. There were significant main effects of size of target test circle $[F(1,11)=23.017, p<.001]$ and size of context circles $[F(2,22)=96.358, p<.001]$, and size of target test circle interacted significantly with display size $[F(2,22)=5.931, p<.020]$ and with size of context circles $[F(2,22)=22.778, p<.001]$. As in Experiments $1,2 \mathrm{~B}$, and 4 , RTs were slower when the observers searched for a small than for a large target test circle (search asymmetry). Although overall RTs were relatively unaffected by display size [no main effect, $F(2,22)=0.840, p=.460]$, there was a slight display size effect on the detection of small target test circles (about $4.5 \mathrm{msec} /$ item) but not on the detection of large target test circles. Furthermore, RTs increased overall with increasing size of context circles, although the increase depended on whether the target test circle was large or small.

This interaction was explored further by two ANOVAs with the factors display size (three, five, or seven) and size of context circles (zero, small, or large), conducted separately for large and small target test circles. There were significant main effects of display size and of size of context circles only with small target test circles $[F(2,22)=$ $4.965, p<.032$, and $F(2,22)=49.121, p<.001$, respectively; for large target test circles, $F(2,22)=2.073$, $p=.176$, and $F(2,22)=1.198, p=.342$, respectively]. Planned comparisons ( $t$ tests) for the small target condition revealed RTs to be slower by approximately $50 \mathrm{msec}$ for test circles surrounded by smaller context circles (inconsistent condition) relative to control conditions for all display sizes $[t(11)=-5.545,-6.339$, and -6.926 , for display sizes of three, five, or seven, respectively; all $p$ s $<.001$ ]; however, there was no RT advantage for consistent relative to control conditions for any display size $[t(11)=1.091,-0.721$, and 0.103 for display sizes of three, five, or seven, respectively]. In contrast to the small target condition, there were no significant effects of size of context circles for the large target condition.

Error analysis. The error rates are presented in Table 6 . An ANOVA of the error data with size of target test circle, display size, target, and size of context circles revealed only a significant display size $\times$ size of context circles interaction $[F(4,44)=4.038, p<.044]$; with large context circles, error rates increased slightly with increasing display size.

\section{Discussion}

The results of Experiment 5 show a pattern similar to that of Experiment 1, in that slower detection RTs (and small display size effects) were found when the observers searched for a small target object among larger distractors than vice versa. This search asymmetry is consistent with the reports from Treisman and Gormican (1988).

The rationale of Experiment 5 was to provide observers with a preview of the context circles (which were assumed to be generally detrimental to search performance), so that they would be able to inhibit these (using a parallel visual-marking process) in advance of the presentation of the target and distractor test circles. If suppression is successful, context circle effects should be reduced or entirely abolished under preview conditions. The pattern of effects in the large target condition conformed with this expectation. The effects produced by the context circles in the no-preview Experiment 1 (the benefits and costs relative to the control condition without context circles) were reduced in magnitude and were no longer statistically significant. In Experiment 1, the consistent condition RTs (small context circle conditions) showed a benefit of $10 \mathrm{msec}$ (combined across display size) relative to the control condition, and the inconsistent condition RTs (large context circle conditions) showed a $22-\mathrm{msec}$ cost. Under preview conditions in Experiment 5, the corresponding figures were 4 and $2 \mathrm{msec}$, respectively. Recall that the observers were not explicitly instructed to suppress the previewed context circles. Thus, the reduction of the context effects in Experiment 5 can be taken to suggest that, in order to detect the target test circle under no-preview conditions, observers tend to suppress the context circles (even though, in principle, they could exploit them to expedite target detection under consistent context conditions). Under no-preview conditions, the context circles would then influence target detection only early during the search on a trial, with their effect diminishing as suppression becomes effective. 
However, the results of the small target condition seem to be at odds with this account, because detection RTs in the inconsistent context condition were longer than those in the control condition despite the preview (in other words, the context circle effect was abolished only in the consistent condition with large context circles, not in the inconsistent condition with small context circles). Indeed, in the no-preview conditions of Experiment 1, both the consistent and inconsistent context conditions showed a cost of $37 \mathrm{msec}$, relative to the control condition, which was taken to mean that the context circles impeded search performance generally and needed to be suppressed to permit successful target detection.

This raises the question of why significant RT costs remained for inconsistent relative to consistent conditions in Experiment 5 (no benefit for consistent conditions, but a 46-msec cost for inconsistent conditions), in which the preview of the context circles was supposed to enable the observers to suppress the context circles in advance of target presentation. A possible answer is that (advance) suppression was possible only under consistent, but not inconsistent, conditions. Under consistent conditions, the target test circle $(6 \mathrm{~mm}$ in diameter) was smaller than both the (larger) context circles $(16 \mathrm{~mm})$ and the distractor test circles $(10 \mathrm{~mm})$, so that suppression could be effectively targeted on large circles (context and distractor test circles). In contrast, under inconsistent conditions, the target test circle $(6 \mathrm{~mm})$ differed little from the (smaller) context circles $(3 \mathrm{~mm})$, and the target size was in between those of the context and distractor test circles (see also Hodsoll, 2002, who demonstrated inefficient search with nonlinearly separable, or intermediate, feature targets in the size dimension). As a result, suppression of the context circles would have tended to affect the target test circle as well, making target detection difficult. ${ }^{4}$

To examine this possibility, a follow-up experiment was carried out, in which the size difference between the target test and context circles was increased from $3 \mathrm{~mm}$ (diameters of the target test circle, the distractor test circles, and the context circles were 6,10 , and $3 \mathrm{~mm}$, respectively, like those in the small target inconsistent condition of Experiment 5) to $6 \mathrm{~mm}$ (diameters of 8, 13, and $2 \mathrm{~mm}$, respectively). It was reasoned that the latter condition would permit better fine tuning of suppression on the context circles (without affecting detectability of the target test circle) and therefore reduce, if not eliminate, the cost for the inconsistent relative to the control condition. Although there was a significant cost reduction, as was expected (from 57 to $36 \mathrm{msec}$ ), the residual cost (of $36 \mathrm{msec}$ ) was still significant. This residual cost, perhaps, points to a general difficulty of suppressing inconsistent (small) context circles when searching for a small target among larger distractors (unfavorable search asymmetry condition).

In summary, except for the small target condition (in which inconsistent context RTs were slower than were consistent context and control condition RTs), the data agree with the idea that the observers were able to exclude the (task-irrelevant) context objects from search and restrict their processing to a (task-relevant) subset of the display items (see also Egeth et al., 1984; Kaptein et al., 1995).

\section{GENERAL DISCUSSION}

Several aspects of the present findings confirm previous studies of visual search for size-defined target objects. If the retinal size difference between the target and distractor objects is sufficiently large, detection RTs are relatively independent of display size, indicating efficient search processes that operate in parallel across the display (e.g., Müller et al., 1995). However, when the size difference between the target and distractor objects is very small, detection RTs increase steeply with increasing display size (with target-present to target-absent slope ratios close to $1: 2$ ), indicating that search involves serial, item-by-item, scanning (Treisman \& Gelade, 1980). Furthermore, there is a search asymmetry in such a way that search for a large target among small distractors is more efficient than search for a small target among large distractors (see Treisman \& Gormican, 1988). ${ }^{5}$

This finding challenges accounts of the search asymmetry for size-defined targets in terms of saliency computations involving parallel comparisons between display items (with targets that are less salient than are distractors not popping out of the display). According to such accounts, no, or at least a reduced, asymmetry would be expected when the search proceeds serially.

More important as to the question at issue, the computation and representation of apparent size, the results of Experiment 1 (and Experiment 3) are clear: Under optimal conditions (i.e., with large targets supporting efficient search), the addition of small context circles to the target and distractor test circles can enhance target detection even relative to the no-context circle control condition, through reinforcing of the retinal size difference between the target and distractor test circles. Under these conditions, the benefits conferred by the context circles are independent of display size (or, if anything, larger at larger display sizes). This supports the idea that the Ebbinghaus apparent size illusion is computed and represented in parallel across the whole display, expediting the computation of target-distractor feature contrast by a given amount, whatever the number of items in the display.

This was the case despite the fact that the addition of context circles to the display was detrimental to performance overall. It was reasoned that the task-irrelevant context circles interfere with the computation of feature contrast between the target and distractor test circles and are therefore suppressed, during the course of a trial, to permit the relevant items to be compared without interference. (Another reason why context circles may need to be suppressed in visual search is that they present ob- 
stacles for implicitly programmed manual grasp movements; see Haffenden, Schiff, \& Goodale, 2001; but see Franz, Bülthoff, \& Fahle, 2003.)

When the search was hard, there were no RT differences dependent on the size of context circles being consistent or inconsistent with the search instruction (search for a large or a small target, respectively), although the addition of context circles to the display, whether consistent or inconsistent, prolonged search, seemingly by an amount relatively independent of display size. In the small target condition, the average costs relative to the control condition were 98, 103, and $118 \mathrm{msec}$ for display sizes of three, five, and seven, respectively (cost increase across display size was $5 \mathrm{msec} /$ item); in the large target condition, the costs were 88,112 , and 138 msec (with a cost increase of $12.5 \mathrm{msec} /$ item). This argues that the benefits (relative to the control condition) produced by the Ebbinghaus apparent size illusion critically depend on conditions supporting efficient search. When search is serial (i.e., not based on parallel computation of saliency contrast between the target and distractor test circles), the illusion does not reinforce the size contrast between the target and distractors, and search proceeds by comparing items individually with a memory representation of the target (or the last item scanned). The fact that the overall cost caused by the addition of context circles (relative to the control condition) is largely additive (independent of display size) might suggest that the context circles are suppressed in a parallel step and that the (serial) search then focuses on test circles with context circles "removed" from the processing (comparison of the test circles with the memory representation, with the memory representation not including context circles).

On the assumption that the context circles impede search overall and need to be suppressed to permit efficient (parallel) comparison between the target and distractor test circles, Experiment 5 tested whether providing observers with a preview of the context circles would permit them to inhibit, or visually mark, the context circles in advance of target and distractor presentation (Olivers, Watson, \& Humphreys, 1999; Watson \& Humphreys, $1997)$, thereby eliminating any consistency effects on detection RTs (see also Cooper \& Weintraub, 1970; Jaeger $\&$ Pollack, 1977). The results largely conformed with this expectation. For the large target condition, which in the no-preview Experiment 1 had produced significant benefits of consistent (small) and significant costs of inconsistent (large) context circles relative to the control condition, the preview afforded in Experiment 5 eliminated all benefits and costs of the context circles. The pattern of effects was less clear-cut for the small target condition, in which the difference between the consistent (large context circles) and the control conditions was eliminated, but the cost for the inconsistent condition (small context circles) was not.

A follow-up experiment confirmed that the latter cost remained, in part because of the small size difference between the target test circle and (in the inconsistent condition) the small context circles, which made it hard to target the inhibition on the context circles without affecting detectability of the target circle. However, this experiment also showed that there remained a significant cost of inconsistent context circles even when the size difference between the target test and context circles was increased. It is not entirely clear what this apparently irreducible cost was caused by. One possibility is a general difficulty of suppressing inconsistent context circles under unfavorable search asymmetry conditions, such as when searching for a small target among larger distractors - that is, when both the target test and context circles are smaller than the distractor test circles and the size of the target test circle is in between those of the context and distractor circles. Possibly, this difficulty arises owing to a natural bias of search within the size dimension toward selecting large templates (Duncan \& Humphreys, 1989) for both the top-down enhancement of target activity and the topdown suppression of distractor activity (see Hodsoll, 2002). ${ }^{6}$

Overall, the present findings are broadly consistent with the idea that the context circles must be suppressed for efficient, parallel, target-distractor discrimination to become possible. On this account, under no-preview conditions, context circle effects arise early after display onset, before inhibition becomes effective.

\section{CONCLUSION}

In summary, the present findings confirm that, at least under favorable search asymmetry conditions, the apparent size of target and distractor objects is derived and compared preattentively in parallel across the display elements. However, although target detection can be facilitated by the context circles modulating the test circles' relative apparent sizes, the context circles also exert a distracting influence on visual search performance and may therefore need to be suppressed. Exactly how the facilitative and interfering effects arising from the context circles play together in determining visual search performance remains to be investigated in further work. One prediction currently under examination is that the overall facilitatory effects of the apparent size modulation depend on the discriminability between the test and context circles, which is influenced by the number of context circles as well as by the distance and lightness contrast between the test and context circles.

\section{REFERENCES}

AKs, D. J., \& EnNs, J. T. (1996). Visual search for size is influenced by a background texture gradient. Journal of Experimental Psychology: Human Perception \& Performance, 22, 1467-1481.

CAve, K. R., \& Wolfe, J. M. (1990). Modeling the role of parallel processing in visual search. Cognitive Psychology, 22, 225-271.

Cooper, L. A., \& Weintraub, D. J. (1970). Delbœuf-type circle illusion: Interactions among luminance, temporal characteristics, and inducingfigure variation. Journal of Experimental Psychology, 85, 75-82.

Coren, S. (1971). A size-contrast illusion without physical size differences. American Journal of Psychology, 84, 565-566.

Duncan, J., \& Humphreys, G. W. (1989). Visual search and stimulus similarity. Psychological Review, 96, 433-458.

Ebbinghaus, H. (1913). Grundzüge der Psychologie. Leipzig: Veit. 
Egeth, H. E., Virzi, R. A., \& Garbart, H. (1984). Searching for conjunctively defined targets. Journal of Experimental Psychology: Human Perception \& Performance, 10, 32-39.

Found, A. (1997). Visual search: Process and representation. Unpublished doctoral thesis, University of London.

Found, A., \& Müller, H. J. (2001). Efficient search for size targets on a background texture gradient: Is detection guided by discontinuities in the retinal-size gradient of items? Perception, 30, 21-48.

Franz, V. H., Bülthoff, H. H., \& Fahle, M. (2003). Grasp effects of the Ebbinghaus illusion: Obstacle avoidance is not the explanation. Experimental Brain Research, 149, 470-477.

HafFenden, A. M., Schiff, K. C., \& Goodale, M. A. (2001). The dissociation between perception and action in the Ebbinghaus illusion: Nonillusory effects of pictorial cues on grasp. Current Biology, 11, 177-181.

HoDSOLL, J. P. (2002). The role of top-down processes in visual search for targets defined by linear and categorical separability. Unpublished doctoral thesis, University of Birmingham, U.K.

Humphreys, G. W., Keulers, N., \& Donnelly, N. (1994). Parallel visual coding in three dimensions. Perception, 23, 453-470.

JAEGer, T., \& Pollack, R. H. (1977). Effect of contrast level and temporal order on the Ebbinghaus circles illusion. Perception \& Psychophysics, 21, 83-87.

Kaptein, N. A., Theeuwes, J., \& van der Heijden, A. H. C. (1995). Search for a conjunctively defined target can be selectively limited to a color-defined subset of elements. Journal of Experimental Psychology: Human Perception \& Performance, 21, 1053-1069.

Massaro, D. W., \& ANDERSON, N. H. (1971). Judgmental model of the Ebbinghaus illusion. Journal of Experimental Psychology, 89, 147-151.

MüLlER, H. J., \& BusCH, A. (2004). Visual search for size-defined target objects is modulated by the Ebbinghaus illusion: Facilitatory and inhibitory effects of the context objects. Manuscript submitted for publication.

MÜller, H. J., Heller, D., \& Ziegler, J. (1995). Visual search for singleton feature targets within and across feature dimensions. Perception \& Psychophysics, 57, 1-17.

Olivers, C. N. L., Watson, D. G., \& Humphreys, G. W. (1999). Visual marking of locations and feature maps: Evidence from withindimension defined conjunctions. Quarterly Journal of Experimental Psychology, 52A, 679-715.

Papathomas, T. V., Feher, A., Julesz, B., \& Zeevi, Y. (1996). Interactions of monocular and cyclopean components and the role of depth in the Ebbinghaus illusion. Perception, 25, 783-795.

PASHLER, H. (1987). Detecting conjunctions of color and form: Reassessing the serial search hypothesis. Perception \& Psychophysics, 41, 191-201.

SEGALOWITZ, S. J., \& Graves, R. E. (1990). Suitability of the IBM XT, $\mathrm{AT}$, and PS/2 keyboard, mouse, and game port as response devices in reaction time paradigms. Behavior Research Methods, Instruments, \& Computers, 22, 283-289.

Treisman, A. (1988). Features and objects: The Fourteenth Bartlett Memorial Lecture. Quarterly Journal of Experimental Psychology, 40A, 201-237.

Treisman, A. M., \& Gelade, G. (1980). A feature-integration theory of attention. Cognitive Psychology, 12, 97-136.

Treisman, A., \& GoRMicAN, S. (1988). Feature analysis in early vision: Evidence from search asymmetries. Psychological Review, 95, 15-48.

Treisman, A., \& Sato, S. (1990). Conjunction search revisited. Journal of Experimental Psychology: Human Perception \& Performance, 16, 459-478.

WATSON, D. G., \& Humphreys, G. W. (1997). Visual marking: Prioritizing selection for new objects by top-down attentional inhibition of old objects. Psychological Review, 104, 90-122.

Wolfe, J. M. (1994). Guided Search 2.0: A revised model of visual search. Psychonomic Bulletin \& Review, 1, 202-238.

Wolfe, J. M. (1998). Visual search. In H. Pashler (Ed.), Attention (pp. 13-73). Hove, U.K.: Psychology Press.

\section{NOTES}

1. Experiment 1 included two further context circle conditions: 6and $10-\mathrm{mm}$ context circles, respectively. Performance under these conditions was similar to the conditions reported here: The search RT functions were flat, with base RTs slower relative to control conditions but faster relative to inconsistent conditions.

2. This means that, for search for the large target, the expected beneficial effect with small relative to large context circles was potentially confounded by the smaller center-to-center distance between the small context circles and the test circles. However, pilot work has shown that search efficiency is overall impeded, rather than enhanced, by smaller distances between test and context circles; that is, potential distance effects would decrease, rather than increase, the context circle effects investigated in Experiment 1. Furthermore, the design of the experiment included a neutral baseline condition (without context circles), permitting potential context circle effects to be tested against the baseline (rather than by directly comparing consistent with inconsistent conditions).

3. Two alternative accounts might attribute the expedited detection of a large target test circle under consistent conditions (with smaller context circles) to depth-based interpretations of the Ebbinghaus configurations and/or lightness contrast between test and context circles. Assuming that all context circles are perceived to lie in the same depth plane, the large target test circle would appear to be nearer than the distractor test circles. Given a bias for near over far targets, this could explain why detection of a large target test circle was expedited under consistent conditions. However, although an account along these lines cannot be ruled out, the contribution of depth-based interpretations was likely to be small with the configurations used in Experiment 1 (Papathomas et al., 1996). Another factor that may have contributed to the expedited detection of large target test circles under consistent conditions is lightness contrast between the test and context circles. Decreasing the lightness contrast increases the apparent size of the test circle (Jaeger \& Pollack, 1977). Taking into account the area of the test and context circles, larger targets surrounded by smaller context circles would exhibit less contrast than would small targets surrounded by larger context circles. This could help to explain why the effect of the Ebbinghaus apparent size illusion was greater in the former condition. However, since there was a strong lightness contrast between the test and context circles in Experiment 1, the influence of this factor on search performance was likely to be small.

4. Under large target conditions in Experiment 5, the size of the target test circle was also in between the sizes of the context and distractor test circles under inconsistent context conditions (context circles, $16 \mathrm{~mm}$; target test circle, $10 \mathrm{~mm}$; distractor test circles, $6 \mathrm{~mm}$ ). However, the size difference between the target test and context circles was larger than that in the inconsistent context small target condition, permitting advance suppression to be better targeted on the context circles.

5. Interestingly, this asymmetry was even more marked (in all conditions, including control conditions) when the search task was hard, likely involving serial scanning of display items. Treisman and Gormican (1988) reported search rates of 14.3 and $7.6 \mathrm{msec} /$ item for smaller and larger targets under easy search conditions and rates of 40.0 and $29.7 \mathrm{msec} /$ item under difficult conditions. That is, the search rate differences were similar.

6. If the preview effects in Experiment 5 are indeed caused by visual marking, the results would suggest that, within the size dimension, marking is applied either to whole feature maps or to spatial regions within maps containing clusters of to-be-suppressed features. This would qualify the proposal of Watson and Humphreys (1997; see also Olivers et al., 1999) that stationary stimuli are marked by inhibition applied to the locations of objects rather than to their features.

(Manuscript received June 1, 2000; revision accepted for publication September 3, 2003.) 